\title{
Depth profiling of noble gas atoms implanted in Al matrix : a photoelectron energy loss spectroscopy study
}

\author{
Christian Godet, ${ }^{1}$ Victor M. da Silva Santana, ${ }^{1,2}$ Denis G.F. David ${ }^{2}$
}

(1) Univ Rennes1, CNRS, IPR (Institut de Physique de Rennes) - UMR 6251, F-35042 Rennes, France

(2) Laboratório de Propriedades Ópticas (LaPO), Instituto de Física, Universidade Federal da Bahia, Campus Universitário de Ondina, 40.210-340 Salvador, Bahia (Brazil).

Keywords : ion implantation, XPS, energy loss, plasmon, depth distribution, noble gas, aluminum

\section{Highlights}

- Non-destructive XPS method provides depth and width of implanted atom profiles

- Measurement of plasmon peak intensities vs photoelectron emission angle

- Analytical model for Gaussian, triangular and rectangular depth distributions

- Model assumes that energy losses are dominated by extrinsic plasmon excitation

- Application to the Ar $\downarrow$ Al system and comparison with Monte Carlo simulations 


\section{ABSTRACT}

A non-destructive spectroscopic method based on core level photoelectron energy loss spectra (PEELS) measured at variable emission angle is proposed for depth profiling of implanted atoms in a solid matrix. Extrinsic plasmon excitation during the photoelectron path to the solid surface being the dominant loss mechanism and neglecting elastic scattering, analytic relations are obtained for several depth profiles (Gaussian, triangular, square) between calculated relative intensities of firstorder and second-order plasmon excitation peaks and the distribution characteristics (average depth and width). This method is illustrated by PEELS analysis of a model system, namely shallow implantation of argon ions $\left(E_{\mathrm{ION}}=2.0 \mathrm{keV}, 35^{\circ}\right.$ incidence, $\left.3 \mathrm{ML}\right)$ into $\mathrm{Al}(001)$ matrix. The average depth $\left(d_{\text {PEELS }}=2.0 \pm 0.1 \mathrm{~nm}\right)$ of subsurface Ar bubbles obtained from energy loss spectra of Ar $2 \mathrm{p}$ photoelectrons (inelastic mean free path $\mathrm{IMFP}=2.15 \mathrm{~nm}$ at $E_{\mathrm{KIN}}=1011 \mathrm{eV}$ ) is significantly smaller than the average depth of the distribution calculated from SRIM code $\left(d_{\text {SRIM }}=3.8 \mathrm{~nm}\right)$. This discrepancy, found for all model depth profiles, is attributed to significant sputtered thickness $(\approx 1.7$ $\mathrm{nm}$ ) of $\mathrm{Al}$, as well as some possible diffusion of argon-vacancy complexes towards the surface during Ar ion implantation. 


\section{Introduction}

Ion interaction with solids has considerable importance in surface physics as analytical probes, in fundamental understanding of irradiation damage mechanisms (e.g. walls of fusion devices) and in device technology for optimizing semiconductor doping profiles and tailoring thin film growth [1-5]. In surface analysis, erosion of solid surfaces by collisional ion beam sputtering is performed to clean samples using chemically inert noble gases (NG) in ultra-high vacuum (UHV) environment [6, 7]. Surface patterning induced by NG ion / solid interactions is well documented in a number of systems $[8,9]$.

The physics of ion impact has been described by a thermal spike model [10, 11], where transient melting of the substrate may be followed by some uncomplete recrystallization. As a consequence, disordered configurations and vacancies may trap implanted atoms and some amount of NG is often retained within the deposited thin film or sputtered metal target (Figure 1). Since noble gas atoms do not form bonds with the metal matrix, precipitation of NG aggregates (clusters, bubbles) is energetically favorable [12-16]. Above some threshold fluence, heavy NG (Ar, Kr, Xe) precipitates are reported to be in the solid phase with epitaxial alignment with the metal matrix [13, 17-19].

Experimental and simulation tools have been developed to understand the complex interplay between displacement and sputtering of target atoms, formation of vacancies and NG-vacancy complexes, NG implantation and nucleation of precipitates [13, 20-24]. As far as the depth profile of embedded NG atoms is concerned, it follows a nearly Gaussian distribution, with steeper outward slope due to the escape of shallow implanted atoms to the vacuum chamber; both projected range (average depth) and straggle (width of the distribution) increase with ion beam energy $E_{\mathrm{ION}}$.

Since typical penetration depth of low-energy $\left(E_{\mathrm{ION}}<5 \mathrm{keV}\right)$ ions is comparable with the inelastic mean free path of photoelectrons [22], recent experiments were focussed on the ability of X-ray photoelectron spectroscopy (XPS) to follow the evolution of NG aggregates; the kinetic energy of core level photoelectrons emitted by embedded atoms being very sensitive to shielding by conduction band electrons of the host material (final state effect), the measured binding energies (BE) give valuable insight in local environment and average bubble size. For the $\operatorname{Ar} \downarrow$ Al model system, it was shown that the bubble diameter increases with ion energy $\left(E_{\mathrm{ION}}\right)$ in the range $1-2 \mathrm{~nm}[\mathbf{1 3 - 1 6 , 2 2 , 2 3 ]}$.

In XPS, core level peak intensity carries little information on the total amount of material as well as on the depth distribution of atoms, in contrast with photoelectron energy losses [25, 26]. On the one hand, some progress has been made recently to obtain depth profiles from zero loss peak (ZLP) 
intensities in angle resolved XPS measurements, however additional stoichiometric constraints are critical for successful recovery of relative concentration profiles [27, 28]. On the other hand, the intensity of the surface and bulk plasmon excitations has been used as a signature of microstructure and material growth mechanisms [29]. In the case of dilute emitter atoms embedded in a solid matrix, besides angular dependence of the host / matrix relative core level peak intensities, used previously [22], in this work complementary information is gained from energy losses experienced by photoelectrons emitted from NG atom core levels along their path through the metal matrix to the sample surface (Figure 1). The excitation of bulk and surface plasmons within the host Al metal has been observed previously for Ar emitters located in subsurface nanobubbles and the bulk plasmon intensity was correlated to $E_{\mathrm{ION}}$ through the average depth distribution of Ar emitters [30].

This Photoelectron Energy Loss Spectroscopy (XPS-PEELS) approach is developed to obtain more quantitative insight in the depth profile of emitter atoms embedded in metal matrix by considering the relative intensities of first-order and second-order plasmon excitation peaks, $\left(I_{1}\left(E_{0}, \alpha\right) / I_{0}\left(E_{0}, \alpha\right)\right)$ and $\left(I_{2}\left(E_{0}, \alpha\right) / I_{0}\left(E_{0}, \alpha\right)\right)$, measured at variable emission angle $\alpha$. The characteristic length scale is $L\left(E_{0}\right)=\lambda\left(E_{0}\right) \cos \alpha$ where the inelastic mean free path (IMFP) related to plasmon excitation, $\lambda\left(E_{0}\right)$, at kinetic energy $E_{0}$ :

$$
\lambda\left(E_{0}\right)^{-1}=\int K\left(E_{0}, \hbar \omega\right) \cdot \mathrm{d} \hbar \omega
$$

is related to the energy loss function (ELF) for electrons travelling through an infinite medium. The differential inverse inelastic mean free path (DIIMFP) is the probability density per unit path length, $K\left(E_{0}, \hbar \omega\right)$, of losing an energy $\hbar \omega$ :

$$
K\left(E_{0}, \hbar \omega\right)=\frac{1}{\pi a_{0} E_{0}} \int_{q^{-}}^{q^{+}} \frac{d q}{q} \operatorname{Im}\left[\frac{-1}{\varepsilon(\omega)}\right]
$$

where $a_{0}$ is the Bohr radius, $q$ is the wave vector transferred from the electron with limit values $\left(q^{-}, q^{+}\right)$ given by energy and momentum conservation during inelastic scattering, and $\varepsilon(\omega)$ is the dielectric function of the solid [31-33].

In this work, extrinsic plasmon excitation during the photoelectron path to the solid surface is assumed to be the dominant loss mechanism, as compared to intrinsic plasmon excitation due to the sudden appearance of the core hole. This result has been established for photoemission from NG nanobubbles in $\mathrm{Al}$ [30]. For the ideal case of a single plane of emitters embedded at depth $d$ in a semiinfinite matrix, simple relations hold for 


$$
\left(I_{1}\left(E_{0}, \alpha\right) / I_{0}\left(E_{0}, \alpha\right)\right)=d / \lambda \cos \alpha \quad\left(I_{2}\left(E_{0}, \alpha\right) / I_{1}\left(E_{0}, \alpha\right)\right)=d / 2 \lambda \cos \alpha .
$$

For real depth profiles, more complex relations are expected between relative intensities of first-order and second-order plasmon excitation peaks and the distribution characteristics, i.e. the normalized depth $(d / \lambda)$ and width $(W / \lambda)$ of the distribution. Analytic relations have been obtained for several depth profiles (Gaussian, triangular, square) considered in Appendix A in order to estimate the robustness of the method and tentatively discriminate physically acceptable model distributions. This work shows that this XPS-PEELS method is weakly sensitive to the exact shape of the distribution, hence the analysis is focused on a Gaussian distribution which matches predictions of the SRIM code (Stopping and Range of Ions in Matter) in homogeneous solids.

This method is evaluated using XPS-PEELS data for the model system $\operatorname{Ar} \downarrow$ Al with low implantation energy $\left(E_{\mathrm{ION}}=2.0 \mathrm{keV}\right)$ and low $\mathrm{Ar}$ ion fluence $(3 \mathrm{ML})$ in a crystalline $\mathrm{Al}(001)$ matrix. Surface preparation, implantation conditions and experimental characterizations are detailed in Section 2. Analysis and modelling of XPS-PEELS data, including the ZLP line shape of Ar 2p core levels and the energy loss function (ELF) of Al metal are reported in Section 3. Some hypotheses proposed for data analysis are discussed in Section 4, and the depth distribution derived from XPS-PEELS is compared with Monte-Carlo based calculations using the SRIM (Stopping and Range of Ions in Matter) code $[\mathbf{1}, \mathbf{3 4}, \mathbf{3 5}]$.

\section{Experimental}

\subsection{Surface preparation}

A crystalline $\mathrm{Al}(001)$ disk $(2 \mathrm{~cm}$ diameter $)$ has been used after extensive sequences of $\mathrm{Ar}$ ion bombardment, resulting in a rough topography. Three successive stages of surface preparation have been characterized by XPS-PEELS: a) oxide-free surface after Ar ion etching (stage \#1), b) slightly contaminated surface after UHV annealing at $500^{\circ} \mathrm{C}$ (2 hours) (stage \#2), c) oxide-free surface after 3 ML Ar ion implantation $\left(E_{\mathrm{ION}}=2.0 \mathrm{keV}\right)$ near room temperature (stage \#3). The surface topography has been imaged after stage \#3 by Scanning Electron Microscopy (JEOL JSM-6610LV) and by Atomic Force Microscopy (SPM 9700, Shimadzu) in the phase mode with a silicon nitride tip.

The argon ion gun (SPECS, IQE 11/35) was operated at $2.0 \mathrm{keV}$ energy with $35^{\circ}$ off-normal incidence; this implantation angle away from channelling directions is expected to enhance the Al sputtering yield per incident argon ion (see section 2.3) [20]. The argon pressure measured in the preparation chamber was adjusted at $7.5 \times 10^{-4} \mathrm{~Pa}$ to obtain full coverage of the substrate holder $(3 \mathrm{~cm}$ 
diameter) by the luminescent $\mathrm{Ar}^{+}$beam cross section $\left(10 \mathrm{~cm}^{2}\right)$. Using a rough estimate of current $\left(5 \times 10^{-}\right.$ $\left.{ }^{6} \mathrm{~A}\right)$, the argon ion flux $\left(3 \times 10^{12}\right.$ ions.cm $\left.{ }^{-2} \cdot \mathrm{s}^{-1}\right)$ provides an ion exposure of $3 \mathrm{ML}\left(3.6 \times 10^{15} \mathrm{Ar}\right.$ ions. $\left.\mathrm{cm}^{-2}\right)$ for 20 minutes irradiation. For the $\mathrm{Al}(001)$ surface, one monolayer (ML) is equivalent to $1.2 \times 10^{15}$ atoms.cm ${ }^{-2}$.

\subsection{XPS analysis}

After each preparation stage, the surface was characterized in situ by XPS under UHV $\left(1.4 \times 10^{-8} \mathrm{~Pa}\right.$ base pressure) using a non-monochromatized X-ray source $(\mathrm{Mg} \mathrm{K} \alpha)$ and photoelectron energy analyzer HAC 100, with constant pass-energy $E_{\text {PASS }}(22 \mathrm{eV}$ for Al 2p core level, $44 \mathrm{eV}$ for Ar 2p core level and survey spectra); respective full width values (1.04 and $1.30 \mathrm{eV})$ of the Gaussian instrumental broadening (including analyzer resolution along with the natural $\mathrm{Mg}$ emission line width $0.7 \mathrm{eV}$ ) were measured on the clean $\mathrm{Al}$ surface. With $\mathrm{Mg} \mathrm{K \alpha}(1253.6 \mathrm{eV})$ excitation, the kinetic energy of $\mathrm{Ar} 2 \mathrm{p}(\mathrm{Al}$ 2p) photoelectrons is $E_{0}=1011 \mathrm{eV}(1183 \mathrm{eV})$ and $\lambda\left(E_{0}\right)=2.15 \mathrm{~nm}(2.43 \mathrm{~nm})$ in the Al matrix [36].

Zero-loss core level peak and energy loss distribution were measured over a broad area $\left(0.3-0.6 \mathrm{~cm}^{2}\right)$ at variable emission angle $\alpha$ between $0^{\circ}$ and $75^{\circ}$ with respect to the surface normal. Before data simulation, the spectra were numerically monochromatized using the main satellites of the Mg source $\left(\mathrm{K} \alpha_{3} / \mathrm{K}_{1,2}=0.08\right.$ and $\left.\mathrm{K} \alpha_{4} / \mathrm{K}_{1 ; 2}=0.04\right)[37,38]$. Mathematical analysis of the Ar $2 \mathrm{p}$ core level peaks was performed using purely Gaussian line profile, with a doublet separation of $2.12 \mathrm{eV}$ between the Ar2p3/2 and Ar2p1/2 peaks. The bulk ELF of the Al matrix was simulated by an asymmetric Lorentzian with half width $\Gamma_{L}=1.6-1.7 \mathrm{eV}$ on the low-energy loss side and half width $\Gamma_{R}=1.8-2.1 \mathrm{eV}$ on the high-energy loss side. Surface plasmon excitations were described by a symmetric Lorentzian with half width $\Gamma_{S 1}=2.1-3.5 \mathrm{eV}$ (first order SP) and $\Gamma_{S 2}=4.6-4.8 \mathrm{eV}$ (second order, due to photoelectrons which have excited one bulk plasmon and one surface excitation). Photoelectrons which have excited two surface plasmons are neglected in the decomposition.

\section{$\underline{2.3}$ SRIM code simulations}

The depth distribution of Ar ions implanted in the $\mathrm{Al}(001)$ target has been obtained using the SRIM2013 (Stopping and Range of Ions in Matter) code [1, 34, 35]. SRIM is a Monte Carlo program that simulates the interactions of energetic ions with amorphous targets. SRIM code calculates energy loss and range of ions into matter using a full quantum mechanical treatment of ion-atom binary collisions. The ion has long range interactions with the target creating electron excitations and plasmons within the target. SRIM code provides the depth distribution of knock-on events, recoiled Al atoms, Al vacancies, 
and final positions of implanted argon atoms, along with the branching ratio for the different energy dissipation channels.

Using default values for the aluminum lattice displacement energy (25 eV) and binding energy (3 $\mathrm{eV})$, about 37.4 vacancies are produced per implanted $\mathrm{Ar}$ ion in our experimental conditions $\left(E_{\mathrm{ION}}=2.0\right.$ $\mathrm{keV}, 35^{\circ}$ incidence). A quasi-Gaussian distribution of $\mathrm{Ar}$ atoms is found with a projected range (average depth) $d_{\text {SRIM }}=3.8 \mathrm{~nm}$, straggling (square root of the variance) $\Delta d=1.9 \mathrm{~nm}$, along with higher order moments (skewness 0.496, kurtosis 3.08). Interestingly, broad distributions of Al recoils and vacancies are found respectively near $2.3 \mathrm{~nm}$ and $2.1 \mathrm{~nm}$, i.e. shallower than the Ar implantation depth, $d_{\text {SRIM }}=3.8 \mathrm{~nm}$.

For further comparison with previous investigations [15], it has been checked that $35^{\circ}$ off-normal ion incidence gives shallower implantation depth $\left(d_{\text {SRIM }}=3.8 \mathrm{~nm}\right)$ and stronger sputtering yield $(Y=$ $2.8)$ as compared with normal ion incidence values $\left(d_{\text {SRIM }}=4.7 \mathrm{~nm}, Y=1.4\right)$.

\section{Results}

\section{$\underline{3.1 \text { Surface topography }}$}

A rough topography of the $\mathrm{Al}(001)$ disk is observed after extensive sequences of $\mathrm{Ar}$ ion bombardment, for a cumulated time of several tens of hours. After exposure to the ambient, SEM images (Figure 2) reveal some cone-shaped craters with an average top diameter of about 2 microns, and a roughened surface with an "orange skin" aspect. In AFM images taken over $2 \mu \mathrm{m}$ x $2 \mu \mathrm{m}$ area (Figure 3), this surface shows some broad undulations or ripples ; in other regions, sharp circular features with micron size diameter were also observed (not shown).

At low fluences used in this work, the Al surface is believed to remain essentially crystalline because Al $2 p$ intensity in angular XPS measurements shows strong photoelectron diffraction effects after all three stages analysed in this work (not shown).

\section{$\underline{3.2 \text { Surface chemistry and binding energies }}$}

Typical oxygen atom coverage in optimized conditions at stages \#1 and \#3 (Figure 4.a) is less than $0.1 \mathrm{ML}\left(8 \times 10^{13} \mathrm{O}\right.$ at. $\left.\mathrm{cm}^{-2}\right)$. At a base pressure of $1.4 \times 10^{-8} \mathrm{~Pa}$, the initial oxidation rate of the clean $\mathrm{Al}$ surface is about $0.01 \mathrm{~nm} /$ hour, as obtained from evolution of the $\mathrm{O}$ 1s peak intensity. After in situ annealing at $500^{\circ} \mathrm{C}$ in UHV (stage \#2), Figure 4.b shows that the Ar 2 p line is completely eliminated, 
as expected from previous reports $[18, \mathbf{1 9}]$, and some contamination attributed to wall outgassing is revealed by $\mathrm{C} 1 \mathrm{~s}, \mathrm{~N} 1 \mathrm{~s}$ and $\mathrm{O} 1 \mathrm{~s}$ peaks in survey spectra.

For all three stages, the matrix core level peaks ( $\mathrm{Al} 2 \mathrm{p}$ and $\mathrm{Al} 2 \mathrm{~s}$ ) do not show significant differences in shape and intensity (less than 1\%); however, a detailed analysis of their loss spectra shows that surface plasmon losses are attenuated at stage \#2 while a slight increase can be detected in the first and second order bulk plasmon peak intensities (Figure 4.c). The zero-loss Al $2 p$ peak (Figure 5) can be well fitted with asymmetric line shape, e.g. with a Doniach-Sunjic shape $[38,39]$ with singularity index $\alpha_{\mathrm{M}}=0.11$ as found previously $[40,41]$. Overall, excellent reproducibility of the multiple order plasmon losses is revealed by the comparison of loss spectra at stages \#1 and \#3.

The binding energies measured on clean $\mathrm{Al}$ surfaces are found at $E_{\mathrm{B}}\left(\mathrm{Al} 2 \mathrm{p}_{3 / 2}\right)=72.85 \pm 0.05 \mathrm{eV}$ and $E_{\mathrm{B}}\left(\operatorname{Ar} 2 \mathrm{p}_{3 / 2}\right)=242.60 \pm 0.05 \mathrm{eV}$ with respect to the Fermi level, giving a difference $E_{\mathrm{B}}(\mathrm{Ar} 2 \mathrm{p})-E_{\mathrm{B}}(\mathrm{Al}$ $2 \mathrm{p})=169.75 \pm 0.10 \mathrm{eV}$. This result will be useful for comparison with previous Ar ion implantation in $\mathrm{Al}(111)$ at normal incidence (section 4) [15, 42].

\section{$\underline{3.3}$ PEELS data analysis}

XPS-PEELS data obtained for the Ar 2p core level being quite similar at stages \#1 and \#3 (Figure 4.b), an angular study was performed in the final stage \#3, i.e. after annealing and ion bombardment at $35^{\circ}$ incidence angle. In the region of $\mathrm{Ar} 2 \mathrm{p}$ and $\mathrm{Ar} 2 \mathrm{~s}$ energy losses (located far from the $\mathrm{Al} 2 \mathrm{~s}$ and $\mathrm{Al}$ 2p lines), a flat background is obtained at stage \#2 (Fig. 4.a and 4.b) after driving out the implanted argon atoms. The plasmon excitation energy, $E_{\mathrm{BP} 1}=15.3 \mathrm{eV}$, is the same for $\mathrm{Al} 2 \mathrm{p}(1183 \mathrm{eV})$ and $\mathrm{Ar}$ 2p (1011 eV) photoelectrons (Figure 5), with a broader distribution in the latter case do to the large spin-orbit splitting. For Ar $2 \mathrm{p}$ photoelectrons, the large relative intensity of plasmon peaks with respect to the zero-loss peak is attributed to a distribution of Ar emitters buried at some average depth $d$, which gives a typical attenuation of the ZLP by a factor $F(d)=\exp \left(-\frac{d}{\lambda \cos \alpha}\right)$,

Zero-loss Ar $2 \mathrm{p}$ peaks obtained in $\mathrm{Ar} \downarrow \mathrm{Al}$ are well fitted with pure Gaussian line shapes, consistent with small values of the singularity index, $\alpha_{\mathrm{M}} \approx 0[43]$ or $\alpha_{\mathrm{M}}<0.02$ found using similar $E_{\mathrm{ION}}=2.0$ $\mathrm{keV},[15]$ as compared with the singularity index found in $\mathrm{Al}\left(\alpha_{\mathrm{M}}=0.10-0.12\right)$ which results from low energy excitations within the large density of states around the Fermi level [38, 40, 41]. This indicates that argon bubbles are large enough for the final state of Ar $2 p$ photoemission to be essentially immune to screening by conduction electrons of the metal matrix. Analysis of the no-loss Ar $2 p$ peaks shows no 
evidence of different components, hence a single dominant environment is considered, i.e. aggregates or bubbles rather than isolated Ar atoms sitting at different matrix defects. The latter would be expected to appear on the negative energy loss side of the ZLP and contribute to make the line shape more asymmetric [15].

Energy loss spectra of Ar 2p photoelectrons are stable at room temperature in UHV between 1 hour and 15 hours after NG implantation. Decomposition of loss spectra is illustrated in Figure 6 for some intermediate emission angle $\left(52^{\circ}\right)$. Since the symmetric line shape of surface plasmon peaks is a rough approximation, the main goal of this decomposition is not a perfect adjustment of experimental data but rather to extract reliable intensity values for $n$-th order $(n=0-2)$ bulk plasmon losses.

At the larger emission angles, some significant increase in the width of first order bulk and surface plasmon ELF is observed, respectively from 3.4 to $3.8 \mathrm{eV}$ and from 4.20 to $6.0 \mathrm{eV}$. The slight increase in BP1 width may arise from the strong approximation of a symmetrical SP1 peak. The significant increase in SP1 width could be related to the large density of internal interfaces (Al / Ar bubble). Although peak adjustment is less reliable for second order losses, a similar increase is also observed, from 3.7 to $4.6 \mathrm{eV}$ in the width of second order bulk plasmon distribution.

As emission angle $\alpha$ increases towards near grazing photoemission, a systematic increase in the relative intensities of bulk plasmon peaks is observed for both first (BP1) and second (BP2) order losses, by a factor of 1.8 and 2.2 respectively, as reported in Table 1. Systematic variations in parameters $d_{1} / \lambda=\cos \alpha\left(I_{1} / I_{0}\right)$ and $d_{2} / \lambda=2 \cos \alpha\left(I_{2} / I_{1}\right)$ confirm that the single plane hypothesis is not valid and reveal a broad distribution width. The surface loss intensity is found to increase faster than the angular dependence $\left(I_{S P 1} \approx 1 / \cos \alpha\right)$ expected for a homogeneous solid surface [33, 40].

\section{$\underline{3.4 \text { Energy loss modeling with Gaussian distribution of embedded emitters }}$}

In order to rationalize the angular dependence of first-order and second-order plasmon loss peak intensities, Appendix A reports calculation results for several depth distributions. The method proposed in this work is illustrated here with a Gaussian depth profile, $G(z)=\left(\pi^{1 / 2} W\right)^{-1} \exp \left[-\left(\frac{d-z}{W}\right)^{2}\right]$, of implanted emitters centered at depth $d$. Using $L\left(E_{0}\right)=\lambda\left(E_{0}\right) \cos \alpha$ and attenuation function $F(z)=\exp \left(-\frac{z}{L}\right)$, the total flux of photoelectrons at kinetic energies $E_{0}, E_{0}-E_{B P 1}$ and $E_{0}-2 E_{B P 1}$, emitted at polar angle $\alpha$ along a rectilinear trajectory is given by 


$$
I_{0}\left(E_{0}, \alpha\right)=\int_{0}^{\infty} d z F(z) G(z), I_{1}\left(E_{0}, \alpha\right)=\int_{0}^{\infty} d z\left(\frac{z}{L}\right) F(z) G(z), I_{2}\left(E_{0}, \alpha\right)=\int_{0}^{\infty} d z\left(\frac{z^{2}}{2 L^{2}}\right) F(z) G(z)
$$

For narrow Gaussian distributions, using Eqn A12-A14, a small straggling parameter $(W<<d)$ yields the approximations:

$$
\begin{aligned}
& I_{1}\left(E_{0}, \alpha\right)=\left(\frac{d}{L}\right)\left[1-\left(\frac{W^{2}}{2 d L}\right)\right] I_{0}\left(E_{0}, \alpha\right) \\
& I_{2}\left(E_{0}, \alpha\right)=\left(\frac{d^{2}}{2 L^{2}}\right)\left[\left(1+\frac{W^{2}}{2 d^{2}}\right)-\left(\frac{W^{2}}{d L}\right)+\left(\frac{W^{4}}{4 d^{2} L^{2}}\right)\right] I_{0}\left(E_{0}, \alpha\right)
\end{aligned}
$$

Since

$$
\left(\frac{I_{1}\left(E_{0}, \alpha\right)}{I_{0}\left(E_{0}, \alpha\right)}\right) \cos (\alpha)=\left(\frac{d}{\lambda}\right)-\left(\frac{W^{2}}{2 \lambda^{2}}\right)(1 / \cos (\alpha))
$$

parameters $(d / \lambda)$ and $(W / \lambda)$ can be graphically obtained from $y$-axis intercept $\left(\frac{d}{\lambda}\right)$ and slope $\left(\frac{-W^{2}}{2 \lambda^{2}}\right)$ in a plot of $\left(\frac{I_{1}\left(E_{0}, \alpha\right)}{I_{0}\left(E_{0}, \alpha\right)}\right) \cos (\alpha)$ vs $(1 / \cos (\alpha))$ (Eqn A19). This narrow distribution limit is represented by red dashed lines in Figure 7.

Otherwise, the full equations (Eqn A12-A14) must be used for parameter adjustment to experimental data. This procedure gives both $d$ and $W$, using tabulated IMFP values $\lambda\left(E_{0}\right)$ of the solid matrix, at the kinetic energy of photoelectrons emitted by embedded Ar atoms. Further check for internal consistency can be obtained from the relative intensities of second order bulk plasmon obtained at different emission angles (Eqn A20), using a plot of $\left(\frac{I_{2}\left(E_{0}, \alpha\right)}{I_{0}\left(E_{0}, \alpha\right)}\right) \cos ^{2}(\alpha)$ vs $(1 / \cos (\alpha))$.

From the experimental data for $\mathrm{Ar} \downarrow \mathrm{Al}$ (Figure 7), we obtain the Gaussian distribution parameters $(d / \lambda)=0.95 \pm 0.05$ and $(W / \lambda)=0.75 \pm 0.05$, which fulfil the hypothesis $(W<d)$. The values of $\left(d_{1} / \lambda\right)$ given by the ideal single plane model, found in the range 0.36-0.79 (Table 1), significantly underestimate the average depth value. Using IMFP values in $\mathrm{Al}, \lambda\left(E_{0}\right)=2.148 \mathrm{~nm}$ at $E_{0}=1011 \mathrm{eV}$, reported by Tanuma et al. [36], we obtain absolute values of $d=2.0 \pm 0.1 \mathrm{~nm}$ and $W=1.6 \pm 0.1 \mathrm{~nm}$ $(\mathrm{FWHM}=2.7 \pm 0.2 \mathrm{~nm})$. In addition, any uncertainty on the IMFP value will also affect error bars on $d$ and $W$ values. 
As discussed further in Section 4, the distribution of implanted Ar ions determined from XPSPEELS data is significantly shallower and broader $\left(d_{\text {PEELS }}=2.0 \pm 0.1 \mathrm{~nm}, \mathrm{FWHM}=2.7 \pm 0.2 \mathrm{~nm}\right)$ than that obtained from SRIM code simulation $\left(d_{\text {SRIM }}=3.8 \mathrm{~nm}, \Delta d=1.9 \mathrm{~nm}\right)$.

\section{Discussion}

This new analytical method based on photoelectron energy loss spectroscopy is expected to be more robust than the usual angular dependence of core level peak intensity to address the depth distribution of implanted atoms; in particular, analysis of bulk plasmon loss intensities gives both the distribution depth and width of implanted atoms in a straightforward way. In this work, the shallow implantation depth of $2 \mathrm{keV} \mathrm{Ar}$ ions in $\mathrm{Al}$ corresponds nearly to the inelastic mean free path, $(d / \lambda)=0.95 \pm 0.05$, of Ar $2 \mathrm{p}$ photoelectrons in $\mathrm{Al}$ using soft $(\mathrm{Mg} \mathrm{K \alpha})$ X-rays. Deeper implantation depth may be better characterized using Hard X-ray Photoelectron Spectroscopy (HAXPES) [44, 45].

In the following, the robustness of the XPS-PEELS method is evaluated (section 4.1) along with implanted argon bubbles characteristics (section 4.2). Energy loss peak intensity is discussed in relation with the physics of plasmon excitation (section 4.3). Possible limitations of the SRIM code and molecular dynamics insigths in Ar atom and vacancy diffusion will be considered to tentatively rationalize the observed discrepancy between PEELS analysis and SRIM simulations (section 4.4).

\section{$\underline{4.1 \text { Robustness of the XPS-PEELS method }}$}

It is well known that the probability for higher order plasmon excitation increases with depth of emitter atom and off-normal emission angle $\alpha$, as observed in this study (Table 1). In contrast, the intensity of the zero-loss peak is strongly attenuated due to plasmon excitation during photoelectron transport towards the surface. The angular dependence of the ZLP intensity is not sufficient to characterize the implanted atom distribution since, for any distribution, the slope of $\log \left(I_{0}\left(E_{0}, \alpha\right)\right)$ vs $1 / \lambda \cos (\alpha)$ depends on distribution width (not shown). Hence both $(d / \lambda)$ and $(W / \lambda)$ parameters need to be determined simultaneously in order to interpret properly core level peak intensity $I_{0}\left(E_{0}, \alpha\right)$ data.

In this work, a graphical analysis is proposed to estimate the deviation from the narrow distribution limit, represented by red dashed lines in Figure 7 ; in our model Ar $\downarrow$ Al system with shallow and broad implanted Ar distribution, this approximation does not hold for most emission angles and the full equations must be used. The distribution width is derived with high precision from the slope 
$\left(-W^{2} / 2 \lambda^{2}\right)$ while the average depth obtained from the y-axis intercept $(d / \lambda)$ is subject to larger uncertainties due to the extrapolation procedure.

In the following, different sources of error are reviewed. Error bar on the implantation depth values produced by uncertainty in the photoelectron IMFP in $\mathrm{Al}$ is about $12 \%$ as estimated from the rootmean-square deviation between IMFP from TPP-2M and IMFP calculated from optical data for aluminum [36].

Elastic scattering increases the photoelectron path length before escape at the solid surface. In XPSPEELS, this corresponds to some enhancement of the plasmon peak relative intensity by a typical amount given by the tabulated ratio between the effective attenuation length and the inelastic mean free path, EAL/IMFP $=0.89$ for $\mathrm{Al}$ at the kinetic energy of $1011 \mathrm{eV} \mathrm{[46]}$. The value of ( $d / \mathrm{IMFP}$ ) derived from modeling of the XPS-PEELS data is thus overestimated by about $10 \%$ if elastic scattering is neglected.

This XPS-PEELS model assumes that the inelastic mean free path for bulk plasmon excitation in $\mathrm{Al}(001)$ is not modified by the presence of Ar bubbles after ion implantation. This assumption is supported by the absence of any change in line-shape and intensity of the Al 2p core-level peaks for the bombarded vs annealed Al surface [14].

SRIM simulations indicate that the distribution of implanted Ar atoms is reasonably approximated by a Gaussian function, with however some skewing towards large implantation depths. In our XPSPEELS model, any deviation from a Gaussian depth distribution is not crucial since very similar plots of first order plasmon loss peak intensity $\left(I_{1} / I_{0}\right) \cos (\alpha)$ as a function of $(1 / \cos (\alpha))$ from quite different (rectangular or triangular) depth distributions, are also adjusted to our data with the same $(d / \lambda)$ value. Different distribution shapes cannot be discriminated unless photoemission is measured at grazing angle with very good signal to noise ratio (Figure 7).

The main difficulty of the method resides in an accurate decomposition of PEELS spectra, since some approximations must be made on bulk and surface plasmon peak shapes to make the problem tractable. For Ar-implanted Al, asymmetric Lorentzian distributions give reasonable description of the ELF shape [37]. At larger emission angle, some significant increase in the width of first order surface plasmon ELF is observed; this could be attributed to the statistical distribution of bubble radii around their mean value, $R$, along with some increased interaction of the photoelectron with several bubbles along its outgoing path. 
In a crystalline solid, the photoelectron flux may be increased along dense atomic rows (focusing) due to many-body coherent elastic scattering; however this forward focusing effect is lost beyond a few atomic distances (defocusing) $[\mathbf{4 7}, \mathbf{4 8}]$. In the $\mathrm{Al}(001)$ matrix, this photoelectron diffraction mechanism modulates the relative intensity of plasmon excitation peak relative to the no-loss $\mathrm{Al} 2 \mathrm{p}$ peak, on the order of 10-20\% (e.g. for $0^{\circ}$ and $45^{\circ}$ directions). Since some epitaxial alignment between solid Ar precipitates and $\mathrm{Al}$ matrix has been reported above a threshold implantation dose, [13, 17-19] some focusing might occur at short distances for Ar $2 p$ photoelectrons but it can be reasonably neglected here because defocusing effects likely dominate for argon bubbles buried at about $2 \mathrm{~nm}$ depth.

\subsection{Argon concentration and bubble size}

In this section, density of implanted argon ions, bubble size and bubble density are estimated in order to check for the validity of this XPS-PEELS analysis. In particular, since formation of very large bubbles of noble gas atoms would change the effective IMFP, this situation would not be consistent with the hypothesis of the XPS-PEELS models developed in Appendix A. It is thus quite important to obtain some calibration of average bubble size and to check that a distribution of few-atom complexes, rather than a few large bubbles, is obtained in our implantation conditions.

In our experiment, a typical value of the apparent Ar content, corrected for photoemission cross sections, gives an atomic ratio $\left[I_{0}(\mathrm{Ar} 2 \mathrm{p}) \sigma(\mathrm{Al} \mathrm{2p}) / I_{0}(\mathrm{Al} 2 \mathrm{p}) \sigma(\mathrm{Ar} 2 \mathrm{p})\right]=0.030 \pm 0.002$. Taking into account the shallower average implantation depth at $35^{\circ}$ incidence, this value is comparable with some values, in the range 0.018 - 0.022, reported for Ar implanted at normal incidence in Al [22, 23]. As a matter of fact, for a buried Gaussian distribution of Ar atoms, a geometrical correction factor, $h(d, W, \alpha, \lambda)=0.342$ at $\alpha=0^{\circ}$, given by Eqn A11, must be applied to obtain the Ar atom density per unit area, $N_{S}(A r)$ :

$$
N_{S}(A r)=\frac{\lambda(A l, 1183 e V)}{h(d, W, \alpha, \lambda)} N_{A T}(A l) \frac{\sigma(A l)}{\sigma(A r)}\left(\frac{I_{0}(A r)}{I_{0}(A l)}\right)
$$

with $\sigma(A l 2 p)=0.11$ and $\sigma\left(\operatorname{Ar} 2 p_{3 / 2}\right)=0.42$. The total Ar concentration, $N_{S}(A r)=1.32 \times 10^{15} \mathrm{Ar}$ atom. $\left.\mathrm{cm}^{-2}\right)$, derived from XPS peak intensity ratio $I_{0}(\mathrm{Ar} 2 \mathrm{p}) / I_{0}(\mathrm{Al} 2 \mathrm{p})$, corresponds to an average density of $0.3 \times 10^{22}$ Ar atom.cm ${ }^{-3}$, taken over a typical depth of $4 \mathrm{~nm} . N_{S}(A r)$ being smaller than the Ar ion fluence $\left(3.6 \times 10^{15} \mathrm{Ar} . \mathrm{cm}^{-2}\right)$, this relatively low retention $(37 \%)$ indicates that some dynamic equilibrium is probably established between ion implantation and exodiffusion of argon atoms. 
Typical argon bubble radius $(R)$ in $\mathrm{Al}$ has been evaluated [15] as a function of the ion implantation energy, $E_{\mathrm{ION}}$, using the variations of the apparent binding energy $E_{B}(\mathrm{Ar} 2 \mathrm{p})$ which results from some shielding of the interaction of Ar $2 p$ photoelectron and Ar $2 p$ positive core hole by conduction band electrons of the host material. Noble gas atoms being less polarizable than the metal matrix, this finalstate contribution to the shift in binding energy comes mostly from the host electronic relaxation upon core ionization of the NG [21]. Assuming a power law increase of bubble radius with ion energy, $R \approx E_{I O N}{ }^{n}$, and considering extra atomic relaxation energy due to conduction electrons of the metal with $(1 / R)$ variation, exponent $n \approx 0.5 \pm 0.2$ was obtained for normal implantation conditions. Absolute $R$ values were derived using the van der Waals Ar atom radius, $R_{0}=0.188 \mathrm{~nm}$, in the limit of isolated Ar atoms, as obtained experimentally at low fluence and low ion energy. In this latter case, the Ar 2p binding energy was $E_{\mathrm{B}}\left(\operatorname{Ar} 2 \mathrm{p}_{3 / 2}\right)=241.95 \mathrm{eV}$ with respect to the Fermi level [15].

For $35^{\circ}$ off-normal implantation $(3 \mathrm{ML})$ at $E_{\mathrm{ION}}=2 \mathrm{keV}$, a much larger binding energy $E_{\mathrm{B}}\left(\operatorname{Ar} 2 \mathrm{p}_{3 / 2}\right)$ $=242.60 \pm 0.05 \mathrm{eV}$ is found, hence the hypothesis of isolated argon atoms can be clearly discarded. Within experimental error, our experimental value compares very well with Biswas et al. results [15] for normal incidence $\mathrm{Ar}$ ion implantation in $\mathrm{Al}(111)$ at the same energy and fluence values, $E_{\mathrm{B}}(\mathrm{Ar} 2 \mathrm{p})=$ $242.65 \mathrm{eV}$, which corresponds to a bubble radius $R \approx 0.48 \mathrm{~nm}$. It is emphasized that the relevant parameter here is ion energy (through the bubble size value as discussed above) rather than fluence value, since for $2 \mathrm{keV}$ argon ions, the steady state BE value is reached for Ar ion fluence of only a fraction of ML [15, 22].

Previous studies of heavy noble gas atom bubbles indicate that, whatever the fluence, the pressure in solid bubbles is always close to the equilibrium pressure [13]; hence in this study, $P_{E} \approx 2 \gamma / R=4.6$ $\mathrm{GPa}$, is estimated from the Al surface tension $\left(\gamma=1.1 \mathrm{~J} . \mathrm{m}^{-2}\right)$ and argon bubble radius $R \approx 0.48 \mathrm{~nm}$. The lattice parameter of solid argon bubbles, deduced previously from diffraction experiments [11], gives a typical molar volume $V_{A r} \approx 19.4 \mathrm{~cm}^{3} \cdot \mathrm{mol}^{-1}$; hence the average number of argon atoms is about 14.4 per bubble, with most argon atoms located at the interface with Al matrix. Neglecting the distribution in bubble radius, a typical nano-bubble density, $N_{\mathrm{B}}(\mathrm{Ar})=2 \times 10^{20}$ bubbles.cm ${ }^{-3}$, is derived from implanted Ar concentration and aggregate size, over some depth of $4 \mathrm{~nm}$, giving a typical inter-bubble distance of $1.7 \mathrm{~nm}$. Similar results were reported previously for low energy Ar ion implantation in Al matrix [30].

In conclusion of this section, the model developed in Appendix A for small subsurface Ar aggregates can be safely used in this XPS-PEELS analysis. Furthermore, since Ar 2p core level reveals 
a single dominant environment with symmetrical line shape, the contribution of isolated Ar atoms in the depth distribution can be neglected.

\section{$\underline{4.3 \text { Physics of plasmon excitation }}$}

This XPS-PEELS model assumes that plasmon excitation by photoelectrons in the host material is essentially extrinsic in nature and its intensity is directly related to the geometrical path of the photoelectron towards the surface. This hypothesis is reasonable for the rather large bubble radius obtained at $E_{\mathrm{ION}}=2.0 \mathrm{keV}$, as shown by the bubble radius dependence of extrinsic and intrinsic plasmon intensities derived by Dhaka et al. [30] but it might not be justified for isolated noble gas atoms embedded in a metal host (as obtained from low fluence and low ion energy implantation), where the intrinsic losses becomes more significant.

A major difference between XPS-PEELS data analysis by different groups lies in the treatment of the spectral background. In line with previous work, [49] we consider that all energy losses above the flat background on the low binding energy side of the ZLP should be assigned to bulk and surface plasmon excitations. In contrast, a broad background subtraction has been imposed [30] on measured Ar $2 p$ energy loss spectra, giving smaller relative intensity, $b\left(\omega_{1 p}\right)$, values of the BP1 peak; at normal emission $\left(\alpha=0^{\circ}\right)$, a scaling factor of about $5 / 3$ would reconcile both data analysis methods, giving here for the decomposition of first order plasmon intensity into its intrinsic and extrinsic components $b\left(\omega_{1 p}\right)=b_{I N T}+b_{E X T} \approx b_{E X T}=0.50 *\left(E_{I O N}\right)^{0.6}\left(b_{I N T}\right.$ being thus negligible for Ar nanobubbles formed at $2 \mathrm{keV})$. Similarly, the intensity ratio between second order and first order bulk plasmon peak intensities is larger in our data analysis, $b\left(\omega_{2 p}\right) / b\left(\omega_{1 p}\right)=0.65 \pm 0.1$, as compared with 0.45 in reference [30].

Interestingly, if we would assume that some inelastic background, distinct from surface or bulk plasmon excitation, must be subtracted from the raw data, giving in first approximation some angle independent scaling factor $S F<1$ for the relative loss intensities, then the graphical representation of angle-dependent relative plasmon intensity (Fig. 7) would provide reduced values of the intercept $(S F d / \lambda)$ and slope $\left(-S F W^{2} / 2 \lambda^{2}\right)$ with two consequences: (i) the decreased $d_{\text {PEELS value would }}$ depart even more from the SRIM simulation $\left(d_{\text {SRIM }}\right)$, (ii) the condition $W<d$ might no longer hold.

As far as the surface plasmon peak (centered at $10.6 \mathrm{eV}$ ) is concerned, our results reveal a strong increase (i.e. faster than the $(1 / \cos \alpha)$ dependence expected for a semi-infinite solid) of its intensity with increasing off-normal emission angle; previous observation of similar effect for $\mathrm{Ne}$ and $\mathrm{Xe}$ subsurface nanobubbles in $\mathrm{Al}$ was attributed to some significant excitation and coupling of bubble 
surface plasmons (BSP) excited at the noble gas bubble / Al matrix interface [30]. The large bubble density and small inter-bubble separation distance found in this work also support this interpretation and may contribute to the large width of surface plasmon excitation peaks.

\subsection{Discrepancy between XPS-PEELS and SRIM implanted depths}

The average depth of argon atoms implanted into $\mathrm{Al}(001)$ matrix $\left(E_{\mathrm{ION}}=2.0 \mathrm{keV}, 35^{\circ}\right.$ off-normal incidence, $3 \mathrm{ML})$ obtained from XPS-PEELS analysis of Ar $2 p$ photoelectrons $\left(d_{\text {PEELS }}=2.0 \pm 0.1 \mathrm{~nm}\right)$ is significantly smaller than that obtained from SRIM code simulation $\left(d_{\text {SRIM }}=3.8 \mathrm{~nm}\right)$. Since the XPSPEELS method robustness was discussed above, we consider here ion implantation modeling issues.

SRIM simulations are very reliable for high energy ion implantation (above $1 \mathrm{keV}$ ) provided that displacement energy is adequately selected. For $2 \mathrm{keV}$ argon ions in Al, SRIM results are comforted by Molecular Dynamics study of ion implantation dynamics, which confirms an average defect depth at $2.0 \mathrm{~nm}$ [50]. In contrast, the relative inaccuracy of SRIM for shallow implantation of noble gases emphasizes the interest of non-destructive analytical tools such as XPS-PEELS, X-ray diffraction, electron microscopy and positron annihilation $[\mathbf{1 2}, \mathbf{1 7}, \mathbf{2 4}, \mathbf{3 0}, \mathbf{5 0}]$ to address depth profiling. However, a limitation of SRIM simulations, as compared with e.g. molecular dynamics, is that a statistical average of cascade phenomena in a virgin target is considered, thus overlooking cumulative effects [51].

The first explanation for the observed discrepancy is due to the large sputtering yield $(Y=2.8)$ for $35^{\circ}$ off-normal implantation of argon ions; about $1.7 \mathrm{~nm}$ of the $\mathrm{Al}$ surface atoms are removed during the overall irradiation process, meaning that the surface is dynamically receding as ion implantation proceeds. Hence, Ar ions implanted at the beginning of the process end up at near-surface locations. Some modification of the Gaussian depth distribution towards a more square-shaped distribution is expected from this dynamical effect, which would account for about $50 \%$ of the discrepancy.

In addition, SRIM is limited to as-implanted damage and neglects any long term and temperature dependent effects such as vacancy and atom diffusion which might affect both distributions. Detailed Molecular Dynamics (MD) studies were recently performed to understand the dynamics of implanted argon atoms and vacancies in Aluminum [52]. In Al, noble gas atoms are unstable at interstitial positions as compared with substitutional positions. Although formation energies for vacancy and divacancy in $\mathrm{Al}, 0.61 \mathrm{eV}$ and $1.38 \mathrm{eV}$, are rather small, out-of-equilibrium mechanisms due to argon ion energy loss are likely dominating near room-temperature, during ion implantation (37 vacancies per 
incident $\mathrm{Ar}$ ion at $2 \mathrm{keV}$, as given by the SRIM code). A small energy barrier, $E_{\mathrm{D}}(\mathrm{V})=0.31 \mathrm{eV}$, has been calculated for vacancy diffusion by jumping to the first neighbor position.

A substitutional Ar atom may act as a sink for mobile free vacancies, to form some Ar-vacancy complex, which could diffuse with a small barrier, $E_{\mathrm{D}}(\mathrm{Ar})=0.85 \mathrm{eV}$ [52]; considering an attempt-toescape frequency on the order of $\mathrm{Al}$ phonon frequency $\left(33.6 \mathrm{meV}, 0.8 \times 10^{13} \mathrm{~s}^{-1}\right)$ [53], migration of argon-vacancy complexes is expected to occur within minutes at room temperature. Hence a possible scenario would be some diffusion of argon-vacancy complexes at ambient temperature, through the vacancy-rich near-surface region, resulting in smaller average depth of final argon atom positions; exodiffusion of argon atoms nearest to the surface and release from the surface to the vacuum is also expected from this scenario.

\section{Conclusion}

A robust analytical method based on photoelectron energy loss spectroscopy (XPS-PEELS) has been developed to obtain both depth and width of implanted atom profiles. This model assumes that plasmon excitation by photoelectrons in the host material is essentially extrinsic (rather than intrinsic) and that the inelastic mean free path is not modified by ion implantation. Simple distribution shapes, addressed in this work, cannot be discriminated unless photoemission is measured at grazing angle with very good experimental signal to noise ratio. Small error bars have been obtained for the model parameters, $(d / \lambda)$ and $(W / \lambda)$, however the accuracy of the absolute depth and width values depends on the uncertainty of the IMFP value for the investigated system and the validity of the rectilinear motion approximation. If the elastic scattering mechanism is not negligible, the correction on depth and width of the profile is given by the ratio (EAL / IMFP).

This method has been illustrated by a model system, namely shallow implantation of low energy argon ions into $\mathrm{Al}(001)$ matrix. Deeper implantation depth may be better characterized using hard Xray photoelectron spectroscopy (HAXPES). In this $\operatorname{Ar} \downarrow A 1$ system, the average depth obtained from PEELS of Ar $2 p$ photoelectrons is significantly smaller than the average depth of the distribution calculated from the Monte Carlo SRIM code. This discrepancy is attributed to a shallow implantation depth $\left(d_{\text {SRIM }}=3.8 \mathrm{~nm}\right)$ at $35^{\circ}$ incidence angle and a significant erosion of surface $\mathrm{Al}$ atoms (thickness $\approx$ $1.7 \mathrm{~nm}$ ) due to the large Al sputtering yield ; additional effects such as some diffusion of argon-vacancy complexes towards the surface during Ar ion implantation deserve more detailed studies. This result 
illustrates the strong complementarity between Monte Carlo or Molecular Dynamics modeling and experimental insight derived from photoelectron energy loss characteristics.

\section{Acknowledgments}

We gratefully acknowledge technical support from Arnaud Le Pottier and Bruno Lépine (IPR, University of Rennes) in the Al sample preparation, along with Tenilson Souza da Silva and Prof. Marcus Vinícius Santos da Silva for SEM and AFM measurements (LAMUME, Universidade Federal da Bahia). One of us (C.G.) is grateful to $\mathrm{CNPq}$ agency for a visiting researcher grant (PVE 400691/2012-4) in the Ciência Sem Fronteiras programme. One of us (D.D.) is grateful to CAPES agency (Brazil) for a senior researcher grant and University of Rennes 1 for an invited professor position. One of us (V.S.S.) is grateful to CAPES agency (Brazil) for PhD grants from CAPES agency (Brazil) and Rennes Métropole.

\# Corresponding author : christian.godet@univ-rennes1.fr 


\section{APPENDIX A - Modelling energy loss peak intensity of photoelectrons emitted by implanted atom distributions}

Photoelectron intensities of the zero-loss peak $\left(I_{0}\right)$, first order $\left(I_{1}\right)$ and second order $\left(I_{2}\right)$ bulk plasmon peaks are derived as a function of emission angle, $\alpha$, for several implantation profiles (Gaussian, rectangular and triangular depth distributions) and compared with an ideal single plane. Exact analytic equations for the relative intensities $\left(I_{1} / I_{0}\right)$ and $\left(I_{2} / I_{0}\right)$ are displayed as a function of $(1 / \cos (\alpha))$ in Figure 7 in order to estimate the departure from the narrow distribution limit which allows graphical determination of relative depth $(d / \lambda)$ and relative width $(W / \lambda)$ parameters.

In this calculation, we assume that plasmon excitation by photoelectrons moving along rectilinear trajectories in the host material is purely extrinsic and that the inelastic mean free path is not modified by the presence of argon bubbles after ion implantation. If the IMFP is the same at kinetic energies $E_{0}, E_{0}-\hbar \omega, E_{0}-2 \hbar \omega$, variation of the outgoing photoelectron fluxes $I_{0}\left(z, E_{0}\right), I_{1}\left(z, E_{0}-\hbar \omega\right)$, $I_{2}\left(z, E_{0}-2 \hbar \omega\right)$ in off-normal direction $\boldsymbol{\alpha}$ through a thin slab of thickness $\mathrm{d} z$ at depth $z$, writes:

$$
\begin{gathered}
\left(\frac{d I_{0}}{d z}\right)=-\frac{I_{0}}{\lambda \cos \alpha} \\
\left(\frac{d I_{1}}{d z}\right)=+\frac{I_{0}}{\lambda \cos \alpha}-\frac{I_{1}}{\lambda \cos \alpha} \\
\left(\frac{d I_{2}}{d z}\right)=+\frac{I_{1}}{\lambda \cos \alpha}-\frac{I_{2}}{\lambda \cos \alpha}
\end{gathered}
$$

Hence,

$$
\begin{aligned}
& I_{1}(z)=\left(\frac{z}{\lambda \cos \alpha}\right) I_{0}(z) \\
& I_{2}(z)=\left(\frac{z}{2 \lambda \cos \alpha}\right) I_{1}(z)
\end{aligned}
$$

The outgoing fluxes depend on the depth distribution of emitter atoms $D(z)$ through:

$$
\begin{aligned}
& I_{0}\left(E_{0}, \alpha\right)=\int_{0}^{\infty} d z F(z) D(z) \\
& I_{1}\left(E_{0}-\hbar \omega, \alpha\right)=\int_{0}^{\infty} d z\left(\frac{z}{L}\right) F(z) D(z) \\
& I_{2}\left(E_{0}-2 \hbar \omega, \alpha\right)=\int_{0}^{\infty} d z\left(\frac{z^{2}}{2 L^{2}}\right) F(z) D(z)
\end{aligned}
$$


where $F(z)=\exp \left(-\frac{z}{L}\right)$ is the attenuation function related to the inelastic mean free path $\lambda\left(E_{0}\right)$, and $L\left(E_{0}\right)=\lambda\left(E_{0}\right) \cos \alpha$. Here photoemission cross section and surface plasmon excitation are omitted.

\section{a) Single plane (Dirac profile)}

For an ideal plane of emitters located at depth $d$, with Dirac profile, $D(z)=\delta(d)$ and unit atom concentration, from Eqns. A4-A5,

$$
I_{0}\left(E_{0}, \alpha\right)=\exp \left(\frac{-d}{\lambda \cos \alpha}\right) \quad \frac{I_{1}\left(E_{0}, \alpha\right)}{I_{0}\left(E_{0}, \alpha\right)}=\left(\frac{d}{\lambda \cos \alpha}\right) \quad \frac{I_{2}\left(E_{0}, \alpha\right)}{I_{1}\left(E_{0}, \alpha\right)}=\left(\frac{d}{2 \lambda \cos \alpha}\right)
$$

\section{b) Gaussian depth distribution}

For a Gaussian density distribution of implanted emitters centered at depth $d$, with full width $F W H M=2 W(\operatorname{Ln} 2)^{1 / 2}$ and unit integrated density:

$$
G(z)=\left(\pi^{1 / 2} W\right)^{-1} \exp \left[-\left(\frac{d-z}{W}\right)^{2}\right]
$$

the analytic expression for $I_{0}\left(E_{0}, \alpha\right)$

$$
I_{0}\left(E_{0}, \alpha\right)=\frac{1}{2} \exp \left(-\frac{d}{L}+\frac{W^{2}}{4 L^{2}}\right)\left[1+\operatorname{erf}\left(\frac{d}{W}-\frac{W}{2 L}\right)\right]
$$

shows that the ZLP intensity decreases (increases) with increasing average depth $d$ (increasing width $W)$ of the Gaussian. The relative intensities $\left(I_{1} / I_{0}\right),\left(I_{2} / I_{1}\right)$ and $\left(I_{2} / I_{0}\right)$ are obtained using the derivative of the Gaussian distribution, $G^{\prime}(z)=2\left(\frac{d-z}{W^{2}}\right) G(z)$ and an integration by parts procedure:

$$
\begin{gathered}
I_{1}\left(E_{0}, \alpha\right)=\left[\left(\frac{d}{L}\right)-\left(\frac{W^{2}}{2 L^{2}}\right)\right] I_{0}\left(E_{0}, \alpha\right)+\pi^{-1 / 2}\left(\frac{W}{2 L}\right) \exp \left[-\left(\frac{d}{W}\right)^{2}\right] \\
I_{2}\left(E_{0}, \alpha\right)=\left(\frac{d}{2 L}\right) I_{1}\left(E_{0}, \alpha\right)+\left(\frac{W^{2}}{4 L^{2}}\right)\left[1-\left(\frac{d}{L}\right)+\left(\frac{W^{2}}{2 L^{2}}\right)\right] I_{0}\left(E_{0}, \alpha\right)-\pi^{-1 / 2}\left(\frac{W^{3}}{8 L^{3}}\right) \exp \left[-\left(\frac{d}{W}\right)^{2}\right]
\end{gathered}
$$

\section{Using Eqn A12,}

$$
I_{2}\left(E_{0}, \alpha\right)=\left[\left(\frac{2 d^{2}+W^{2}}{4 L^{2}}\right)-\left(\frac{d W^{2}}{2 L^{3}}\right)+\left(\frac{W^{4}}{8 L^{4}}\right)\right] I_{0}\left(E_{0}, \alpha\right)+\pi^{-1 / 2}\left(\frac{W d}{4 L^{2}}-\frac{W^{3}}{8 L^{3}}\right) \exp \left[-\left(\frac{d}{W}\right)^{2}\right]
$$


For small Gaussian width ( $W<d$ ), and $\alpha$ not too large, the last exponential term in Eqns. 12, 13, 14 is negligible, hence:

$$
\begin{gathered}
I_{1}\left(E_{0}, \alpha\right)=\left(\frac{d}{L}\right)\left[1-\left(\frac{W^{2}}{2 d L}\right)\right] I_{0}\left(E_{0}, \alpha\right) \\
I_{2}\left(E_{0}, \alpha\right)=\left(\frac{d}{2 L}\right) I_{1}\left(E_{0}, \alpha\right)+\left(\frac{W^{2}}{4 L^{2}}\right)\left[1-\left(\frac{d}{L}\right)+\left(\frac{W^{2}}{2 L^{2}}\right)\right] I_{0}\left(E_{0}, \alpha\right)
\end{gathered}
$$

Inserting Eqn A15 into Eqn A16, exact analytic formulae are obtained:

$$
\begin{gathered}
I_{2}\left(E_{0}, \alpha\right)=\left(\frac{d^{2}}{2 L^{2}}\right)\left[\left(1+\frac{W^{2}}{2 d^{2}}\right)-\left(\frac{W^{2}}{d L}\right)+\left(\frac{W^{4}}{4 d^{2} L^{2}}\right)\right] I_{0}\left(E_{0}, \alpha\right) \\
I_{2}\left(E_{0}, \alpha\right)=\left(\frac{d}{2 L}\right)\left[1+\frac{\left(\left(\frac{L}{d}-1\right) W^{2}+\frac{W^{4}}{2 d L}\right)}{\left(2 d L-W^{2}\right)}\right] I_{1}\left(E_{0}, \alpha\right)
\end{gathered}
$$

Eqn A9 is recovered in the narrow distribution approximation $(W \rightarrow 0)$. As expected, Eqn A15 shows that the relative intensity $\frac{I_{1}\left(E_{0}, \alpha\right)}{I_{0}\left(E_{0}, \alpha\right)}$ of the first bulk plamon peak is reduced with increasing width of the Gaussian distribution. However, Eqn A18 reveals that the relative intensity $\frac{I_{2}\left(E_{0}, \alpha\right)}{I_{1}\left(E_{0}, \alpha\right)}$ may be either increased $(d<\lambda \cos \alpha)$ or decreased $(d>\lambda \cos \alpha)$ by the effect of the distribution width, depending on the value of the implantation depth, $d$, relative to the inelastic mean free path. In addition, the ratio $\frac{I_{2}\left(E_{0}, \alpha\right)}{I_{1}\left(E_{0}, \alpha\right)} \cos (\alpha)$ is expected to decrease with increasing (grazing) emission angles.

For a small width of the Gaussian profile, plotting first order plasmon loss peak relative intensity times $\cos (\alpha)$ as a function of $(1 / \cos (\alpha))$ gives a linear dependence, in the narrow distribution approximation:

$$
\left(\frac{I_{1}\left(E_{0}, \alpha\right)}{I_{0}\left(E_{0}, \alpha\right)}\right) \cos (\alpha)=\left(\frac{d}{\lambda}\right)-\left(\frac{W^{2}}{2 \lambda^{2}}\right)(1 / \cos (\alpha))
$$


Graphical determination of $y$-axis intercept $\left(\frac{d}{\lambda}\right)$ and slope $\left(\frac{-W^{2}}{2 \lambda^{2}}\right)$ yields the Gaussian distribution parameters provided that the IMFP, $\lambda$, is known for core level photoelectrons of implanted atoms travelling through the matrix.

Further check for internal consistency can be obtained from the second order bulk plasmon loss peak intensity times $\cos ^{2}(\alpha)$ obtained at different emission angles. For a narrow Gaussian profile, plotting angular results for second order plasmon losses should also give a linear dependence as a function of $(1 / \cos (\alpha))$ :

$$
\left(\frac{I_{2}\left(E_{0}, \alpha\right)}{I_{0}\left(E_{0}, \alpha\right)}\right) \cos ^{2}(\alpha) \approx\left(\frac{2 d^{2}+W^{2}}{4 \lambda^{2}}\right)-\left(\frac{d W^{2}}{2 \lambda^{3}}\right)(1 / \cos (\alpha))
$$

This plot using the relative intensity of second order bulk plasmon allows to compare the measured yaxis intercept $\left(\frac{2 d^{2}+W^{2}}{4 \lambda^{2}}\right)$ and slope $\left(-\frac{d W^{2}}{2 \lambda^{3}}\right)+\left(\frac{W^{4}}{8 \lambda^{4} \cos \alpha}\right) \approx\left(-\frac{d W^{2}}{2 \lambda^{3}}\right)$, with the respective values calculated using previously determined $(d / \lambda)$ and $(W / \lambda)$ parameters.

\section{c) Triangular depth distribution}

For a symmetrical triangular distribution buried between depths $(d-V)$ and $(d+V)$ with unit area (height $1 / V$ ), integration by parts gives exact analytic formula:

$$
\begin{gathered}
I_{0}\left(E_{0}, \alpha\right)=\exp \left(-\frac{d}{L}\right)\left(\frac{2 L^{2}}{V^{2}}\right)\left[-1+\cosh \left(\frac{V}{L}\right)\right] \\
I_{1}\left(E_{0}, \alpha\right)=\exp \left(-\frac{d}{L}\right)\left(\frac{2 L}{V}\right)\left\{\frac{1}{V}(2 L+d)\left(-1+\cosh \left(\frac{V}{L}\right)\right)-\sinh \left(\frac{V}{L}\right)\right\} \\
I_{2}\left(E_{0}, \alpha\right)=\exp \left(-\frac{d}{L}\right)\left\{\frac{1}{V^{2}}\left(6 L^{2}+4 L d+d^{2}\right)\left(-1+\cosh \left(\frac{V}{L}\right)\right)+\cosh \left(\frac{V}{L}\right)-\frac{2}{V}(d+2 L) \sinh \left(\frac{V}{L}\right)\right\}
\end{gathered}
$$

with power law expressions in the narrow distribution approximation $(V<<L)$ :

$$
I_{0}\left(E_{0}, \alpha\right) \approx V \exp \left(-\frac{d}{L}\right)\left(1+\frac{V^{2}}{12 L^{2}}\right)
$$




$$
\begin{gathered}
\frac{I_{1}\left(E_{0}, \alpha\right)}{I_{0}\left(E_{0}, \alpha\right)} \approx\left(\frac{d}{L}\right)\left[1-\left(\frac{V^{2}}{3 L d}\right)\right] \\
\frac{I_{2}\left(E_{0}, \alpha\right)}{I_{0}\left(E_{0}, \alpha\right)} \approx\left(\frac{d^{2}}{2 L^{2}}\right)\left[1-\left(\frac{V^{2}}{3 d^{2}}\right)-\left(\frac{2 V^{2}}{3 L d}\right)\right]
\end{gathered}
$$

\section{d) Rectangular depth distribution}

For a rectangular distribution buried between depths $(d-U)$ and $(d+U)$ with unit area (height $1 / 2 U$ ) and width $2 U$, integration by parts gives exact analytic formula:

$$
\begin{gathered}
I_{0}\left(E_{0}, \alpha\right)=\left(\frac{L}{U}\right) \exp \left(-\frac{d}{L}\right) \sinh \left(\frac{U}{L}\right) \\
\frac{I_{1}\left(E_{0}, \alpha\right)}{I_{0}\left(E_{0}, \alpha\right)}=\left(\frac{d+L}{L}\right)-\left(\frac{U}{L}\right) \operatorname{coth}\left(\frac{U}{L}\right) \\
\frac{I_{2}\left(E_{0}, \alpha\right)}{I_{0}\left(E_{0}, \alpha\right)}=\left(\frac{d+L}{L}+\frac{d^{2}+U^{2}}{2 L^{2}}\right)-\left(\frac{U}{L}\right)\left(1+\frac{d}{L}\right) \operatorname{coth}\left(\frac{U}{L}\right)
\end{gathered}
$$

with power law expressions in the narrow distribution approximation $(U<<L)$ :

$$
\begin{gathered}
I_{0}\left(E_{0}, \alpha\right) \approx \exp \left(-\frac{d}{L}\right)\left(1+\frac{U^{2}}{6 L^{2}}\right) \\
\frac{I_{1}\left(E_{0}, \alpha\right)}{I_{0}\left(E_{0}, \alpha\right)} \approx\left(\frac{d}{L}\right)\left(1-\frac{U^{2}}{3 L d}\right) \\
\frac{I_{2}\left(E_{0}, \alpha\right)}{I_{0}\left(E_{0}, \alpha\right)} \approx\left(\frac{d^{2}}{2 L^{2}}\right)\left[1+\frac{U^{2}}{3 d^{2}}-\frac{2 U^{2}}{3 L d}\right]
\end{gathered}
$$




\section{REFERENCES}

[1] J.F. Ziegler, J.P. Biersack, U. Littmark, The Stopping and Range of Ions in Solids, Pergamon, New York, 1985.

[2] R. Behrisch (ed.), Sputtering by Particle Bombardment I: Physical Sputtering of Single-Element Solids (Springer, New York, 1981).

[3] J.J. Cuomo, S.M. Rossnagel, H.R. Kaufman (eds.): Handbook of Ion Beam Processing Technology (Noyes, Park Ridge 1989).

[4] J.S. Williams, Ion implantation in semiconductors, Mat. Sci. Eng. A 253 (1998) 8-15.

[5] T. Itoh (ed.): Ion Beam Assisted Film Growth, Elsevier, Amsterdam 1989.

[6] G.P. Chambers, J. Fine, Pure element sputtering yield data, in: D. Briggs, M.P. Seah (Eds.), Practical Surface Analysis, Ion and Neutral Spectroscopy, 2nd ed., vol. 2, Wiley, New York, 1992, p. 705.

[7] T. Akita, T. Nagata, Y. Kimura, Y. Takai, R. Shimizu, Observation of Al surface during sputter-cleaning and annealing procedures under UHV-REM. J Electron Microsc (Tokyo) 47 (1998) 223-226.

[8] J. Muñoz-García, L. Vazquez, M. Castro, R. Gago, A.R. Cubero, A. M. Barrado, R. Cuerno, Selforganized nanopatterning of silicon surfaces by ion beam sputtering, Mat. Sci. Eng. R 86 (2014) 1-44.

[9] S. Cucatti, E.A. Ochoa, M. Morales, R. Droppa Jr., J. Garcia, H.C. Pinto, L.F. Zagonel, D. Wisnivesky, C.A. Figueroa, F. Alvarez, Effect of bombarding steel with Xe+ ions on the surface nanostructure and on pulsed plasma nitriding process, Mater. Chem. Phys. 149-150 (2015) 261-269.

[10] M. Toulemonde, E. Paumier, C. Dufour, Thermal spike model in the electronic stopping power regime, Radiation Effects Defects in Solids 126 (1993) 201-206.

[11] H. Hofsäss, H. Feldermann, R. Merk, M. Sebastian, C. Ronning, Cylindrical spike model for the formation of diamondlike thin films by ion deposition, Applied Physics A 66 (1998) 153-181.

[12] A.K. Tyagi, R. Khanna, G.V.N. Rao, Solid argon bubbles in aluminium, Scripta Met. 20 (1986) 12451246.

[13] C. Templier, Inert Gas Bubbles in Metals: A Review. In: S.E. Donnelly, J.H. Evans (eds) Fundamental Aspects of Inert Gases in Solids. NATO ASI Series (Series B: Physics), vol. 279, Springer, Boston, MA, 1991, pp. 117-132.

[14] C. Biswas, A.K. Shukla, S. Banik, V.K. Ahire, S.R. Barman, XPS and LEED study of Argon bombarded Al(111) surface, Nucl. Instrum. Meth. Phys. Res. B 212 (2003) 297-302.

[15] C. Biswas, A.K. Shukla, S. Banik, S.R. Barman, A. Chakrabarti, Argon Nanobubbles in Al(111): A Photoemission Study, Phys. Rev. Lett. 92 (2004) 115506.

[16] A.R. Lahrood, T. de los Arcos, M. Prenzel, A. von Keudell, J. Winter, X-ray photoelectron spectroscopy on implanted argon as a tool to follow local structural changes in thin films, Thin Solid Films 520 (2011) 1625-1630.

[17] S.E. Donnelly, C.J. Rossouw, Lattice images of inert gas bubbles in aluminum, Nucl. Instrum. Meth. Phys. Res. B 13 (1986) 485-489.

[18] R.C. Birtcher, W. Jager, Precipitation of $\mathrm{Kr}$ after implantation into Al, Nucl. Instrum. Meth. Phys. Res. B 15 (1986) 435-438.

[19] R.J. Cox, P.J. Goodhew, J.H. Evans, A study of the solidification of argon bubbles in aluminum, Acta Metall. 35 (1987) 2497-2501. 
[20] S. Valkealahti, R.M. Nieminen, Molecular dynamics simulation of the damage production in $\mathrm{Al}(110)$ surface with slow argon ions, Nucl. Instrum. Meth. Phys. Res. B 18 (1987) 365-369.

[21] R.G. Lacerda, M.C. dos Santos, L.R. Tessler, P. Hammer, F. Alvarez, F.C. Marques, Pressure-induced physical changes of noble gases implanted in highly stressed amorphous carbon films, Phys. Rev. B 68 (2003) 4104.

[22] R.S. Dhaka, C. Biswas, A.K. Shukla, S.R. Barman, A. Chakrabarti, Xe and Ar nanobubbles in Al studied by photoemission spectroscopy, Phys. Rev. B 77 (2008) 104119.

[23] R.S. Dhaka, S.R. Barman, X-ray photoemission studies on rare gas bubbles in aluminium with annealing temperature, Surf. Coat. Technol. 203 (2009) 2380-2382.

[24] R.S. Dhaka, K. Gururaj, S. Abhaya, G. Amarendra, S. Amirthapandian, B.K. Panigrahi, K.G.M. Nair, N.P. Lalla, S.R. Barman, Depth-resolved positron annihilation studies of argon nanobubbles in aluminum, J. Appl. Phys. 105 (2009) 054304.

[25] D. Briggs, M.P. Seah, Practical Surface Analysis, Vol. 1, Auger and X-ray Photoelectron Spectroscopy, second ed., John Wiley and Sons, 1990.

[26] S. Hajati, S. Coultas, C. Blomfield, S. Tougaard, XPS imaging of depth profiles and amount of substance based on Tougaard's algorithm, Surf. Sci. 600 (2006) 3015-3021.

[27] M. Szklarczyk, K. Macak, A.J. Roberts, K. Takahashi, S. Hutton, R. Głaszczka, C. Blomfield, Subnanometer resolution XPS depth profiling: sensing of atoms, Appl. Surf. Sci. 411 (2017) 386-393.

[28] A.V. Lubenchenko, A.A. Batrakov, A.B. Pavolotsky, O.I. Lubenchenko, D.A. Ivanov, XPS study of multilayer multicomponent films, Appl. Surf. Sci. 427 (2018) 711-721.

[29] J.P. Holgado, A. Barranco, F. Yubero, J.P. Espinos, A.R. Gonzalez-Elipe, Surface microstructure of MgO deposited on $\mathrm{SiO} 2$ by analysis of plasmon excitations in photoemission experiments, Surf. Sci. 482 (2001) 1325-1330.

[30] R.S. Dhaka, S.R. Barman, Plasmon Excitations by Photoelectron Emission from Rare Gas Nanobubbles in Aluminum, Phys. Rev. Lett. 104 (2010) 036803.

[31] W.S.M. Werner, Electron transport in solids for quantitative surface analysis, Surf. Interface Anal. 31 (2001) 141-176.

[32] F. Yubero, S. Tougaard, Quantification of plasmon excitations in core-level photoemission, Phys. Rev. B 71 (2005) 045414.

[33] D. David, C. Godet, Derivation of dielectric function and inelastic mean free path from photoelectron energy-loss spectra of amorphous carbon surfaces, Appl. Surf. Sci. 387 (2016) 1125-1139.

[34] J.P. Biersack, L.G. Haggmark, A Monte Carlo computer program for the transport of energetic ions in amorphous targets, Nucl. Instrum. Methods 174 (1980) 257-269. https://doi.org/NUIMAL

[35] J. F. Ziegler, SRIM-2013 software package, see http://www.srim.org.

[36] S. Tanuma, C.J. Powell, D.R. Penn, Calculations of electron inelastic mean free paths. VIII. Data for 15 elemental solids over the 50-2000 eV range, Surf. Interf. Anal. 36 (2004) 1-14.

[37] C. Klauber, Magnesium Ka X-ray line structure revisited, Appl. Surf. Sci. 70/71 (1993) 35-39.

[38] V.M. Santana, D. David, J.S. de Almeida, C. Godet, Photoelectron energy-loss in Al(002) revisited : retrieval of the single plasmon loss energy distribution by a Fourier transform method, Braz. J. Phys. 48 (2018) 215-226. doi: 10.1007/s13538-018-0566-8

[39] S. Doniach, M. Sunjic, Many-electron singularity in x-ray photoemission and-ray line spectra from metals. J. Phys. C3 (1970) 285-291. 
[40] S.Hüfner, Photoelectron Spectroscopy: principles and applications, Springer-Verlag, Berlin, 2003.

[41] P.H. Citrin, G.K. Wertheim, Y. Baer, Many-body processes in x-ray photoemission line shapes from Li, $\mathrm{Na}, \mathrm{Mg}$, and Al metals, Phys. Rev. B 16 (1977) 4256.

[42] C. Biswas, A.K. Shukla, S. Banik, V.K. Ahire, S.R. Barman, Plasmons in core-level photoemission spectra of Al(111), Phys. Rev. B 67 (2003) 165416.

[43] M. Abbate, Line asymmetry in the X-ray photoelectron spectra of Ar and K implanted in Al, Phys. Rev. B 39 (1989) 7641-7644.

[44] P. Risterucci, O. Renault, E. Martinez, B. Detlefs, V. Delaye, J. Zegenhagen, C. Gaumer, G. Grenet, S. Tougaard, Probing deeper by hard x-ray photoelectron spectroscopy, Appl. Phys. Lett. 104 (2004) 051608.

[45] F. Offi, W.S.M. Werner, M. Sacchi, P. Torelli, M. Cautero, G. Cautero, A. Fondacaro, S. Huotari, G. Monaco, G. Paolicelli, W. Smekal, G. Stefani, G. Panaccione, Comparison of hard and soft x-ray photoelectron spectra of silicon, Phys. Rev. B 76 (2007) 085422.

[46] A. Jablonski, C. Powell, Practical expressions for the mean escape depth, the information depth, and the effective attenuation length in Auger-electron spectroscopy and x-ray photoelectron spectroscopy, J. Vac. Sci. Technol. A 27 (2009) 253-261.

[47] M. Kazama, H. Shinotsuka, Y. Ohori, K. Niki, T. Fujikawa, L. Kover, Plasmon losses in core-level photoemission spectra studied by the quantum Landau formula including full multiple scattering, Phys. Rev. B 89 (2014) 045110.

[48] J. Osterwalder, T. Greber, S. Hufner, L. Schlapbach, Photoelectron diffraction from core levels and plasmon-loss peaks of aluminum, Phys. Rev. B 41 (1990) 12495.

[49] W.J. Pardee, G.D. Mahan, D.E. Eastman, R.A. Pollak, L. Ley, F.R. McFeely, S.P. Kowalczyk, D.A. Shirley, Analysis of surface- and bulk-plasmon contributions to X-ray photoemission spectra, Phys. Rev. B 11 (1975) 3614-3616.

[50] A. Vehanen, J. Makinen, P. Hautojarvi, H. Huomo, J. Lahtinen, R.M. Nieminen, S. Valkealahti, Nearsurface defect profiling with slow positrons: Argon-sputtered Al(110), Phys. Rev. B 32 (1985) 7561.

[51] L. Pentecoste, P. Brault, A.L Thomann, P. Desgardin, T. Lecas, T. Belhabib, M.F. Barthe, T. Sauvage, Low Energy and low fluence helium implantations in tungsten: Molecular dynamics simulations and experiments. J. Nucl. Materials 470 (2016) 44-54.

[52] X. Gai, Radiation damage and inert gas bubbles in metals, PhD thesis, Loughborough University, 2015. https://dspace.lboro.ac.uk/2134/17927 (accessed 07/09/2017).

[53] C.Y. Ho, R.W. Powell, P.E. Liley, Thermal Conductivity of the Elements: A Comprehensive Review, J. Phys. Chem. Ref. Data 3 (1974) 1-796. 
Table 1 : Decomposition of Ar 2p photoelectron energy loss spectra for Argon nanobubbles embedded in Al metal matrix. Typical error bars were estimated at $\pm 1 \%$ for ZLP area, $\pm 10 \%$ for BP1 area, $\pm 25 \%$ for BP2 area. Relative intensities of first order and second order bulk plasmon peaks (normalized to ZLP area) were used to calculate apparent emitter depths, $d_{1} / \lambda=\cos \alpha\left(I_{1} / I_{0}\right)$ and $d_{2} / \lambda=2 \cos \alpha\left(I_{2} / I_{1}\right)$, within the ideal single plane model (columns 5 and 6$)$. The last column shows large surface plasmon intensity at $65^{\circ}$ emission angle.

\begin{tabular}{|c|c|c|c|c|c|c|}
\hline $\begin{array}{c}\text { Angle } \\
\text { (degree) }\end{array}$ & $\begin{array}{c}\text { ZLP area } \\
\text { (a.u.) }\end{array}$ & $\begin{array}{c}\text { BP1 area } \\
\text { (a.u.) }\end{array}$ & $\begin{array}{c}\text { BP2 area } \\
\text { (a.u.) }\end{array}$ & $\begin{array}{c}\cos \alpha\left(I_{1} / I_{0}\right) \\
2 \cos \alpha\left(I_{2} / I_{1}\right)\end{array}$ & $\begin{array}{c}\cos \alpha \times I_{S P 1} \\
\text { (a.u.) }\end{array}$ \\
\hline 0 & 1 & 0.786 & 0.470 & 0.79 & 1.20 & 0.26 \\
\hline 52 & 1 & 1.01 & 0.708 & 0.62 & 0.87 & 0.28 \\
\hline 65 & 1 & 1.39 & 1.04 & 0.59 & 0.63 & 0.45 \\
\hline 75 & 1 & 1.39 & 1.04 & 0.36 & 0.39 & 0.26 \\
\hline
\end{tabular}




\section{FIGURE CAPTIONS}

Figure 1: Schematic representation of subsurface noble gas precipitates (nanobubbles). Photoelectrons produced by X-ray photoionization of $\mathrm{Ar} 2 \mathrm{p}$ core levels experience energy losses due to bulk and surface plasmon excitation, as they cross the Al matrix towards the surface. Both apparent ZLP binding energy and plasmon peak intensities are influenced by the geometrical parameters (depth and width of the distribution, average bubble radius) of the argon precipitates.

Figure 2: Scanning electron microscope image (x 15000) of the $\mathrm{Al}(002)$ surface after extensive Ar ion bombardment sequences showing an "orange skin" aspect and 2 micron width craters.

Figure 3: Contact-mode AFM image $\left(2 \times 2 \mu \mathrm{m}^{2}\right)$ of the $\mathrm{Al}$ surface (color code height $\left.3.1 \mathrm{~nm}\right)$.

Figure 4: XPS characterization at normal emission angle $\left(\alpha=0^{\circ}\right)$ of the $\mathrm{Al}(001)$ surface at stage \#1 (Ar-implanted), stage \#2 (annealed) and stage \#3 (Ar implanted, 3 ML) : a) Survey spectra, b) Ar 2s, Ar $2 \mathrm{p}$ and $\mathrm{C} 1 \mathrm{~s}$ peaks, c) Al $2 \mathrm{p}$ core level and multiple plasmon excitation peaks. The Al surface is oxygen-free at stages \#1 and \#3.

Figure 5: XPS-PEELS at variable emission angle $\left(\alpha=0^{\circ}, 52^{\circ}, 65^{\circ}\right)$ of Ar $2 p$ core level and multiple plasmon excitation peaks (peak labeling, see Fig. 6). Reference spectrum for plasmon excitation by Al $2 \mathrm{p}$ photoelectrons was obtained at normal emission $\left(\alpha=0^{\circ}\right)$ and smaller instrument broadening.

Figure 6: Decomposition of XPS-PEELS data for the Ar 2p core level at $\alpha=52^{\circ}$, into first order and second order bulk and surface plasmon peaks. Peak BP2 $(30.8 \mathrm{eV})$ is due to photoelectrons which have excited two bulk plasmons. Peak SP2 $(25.7 \mathrm{eV})$ is due to photoelectrons which have excited one bulk plasmon and one surface excitation. Photoelectrons which have excited two surface plasmons are neglected in the decomposition.

Figure 7: Emission angle dependence of the relative intensities of first-order (a) and second-order (b) plasmon peaks for $\operatorname{Ar} \downarrow \mathrm{Al}\left(E_{\mathrm{ION}}=2.0 \mathrm{keV}, 35^{\circ}\right.$ incidence, $3 \mathrm{ML}$ ). Using the full equations (Appendix A) for Gaussian $(\mathrm{G})$, Rectangular $(\mathrm{R})$ and Triangular $(\mathrm{T})$ depth distributions, the average depth and width are adjusted to the data for each model depth distribution (inset). The influence of distribution width $(W)$ is evidenced by the departure of data from the red dashed line, obtained by injecting the adjusted depth and width values into the narrow Gaussian limit (Eqn 5). 
Figure 1

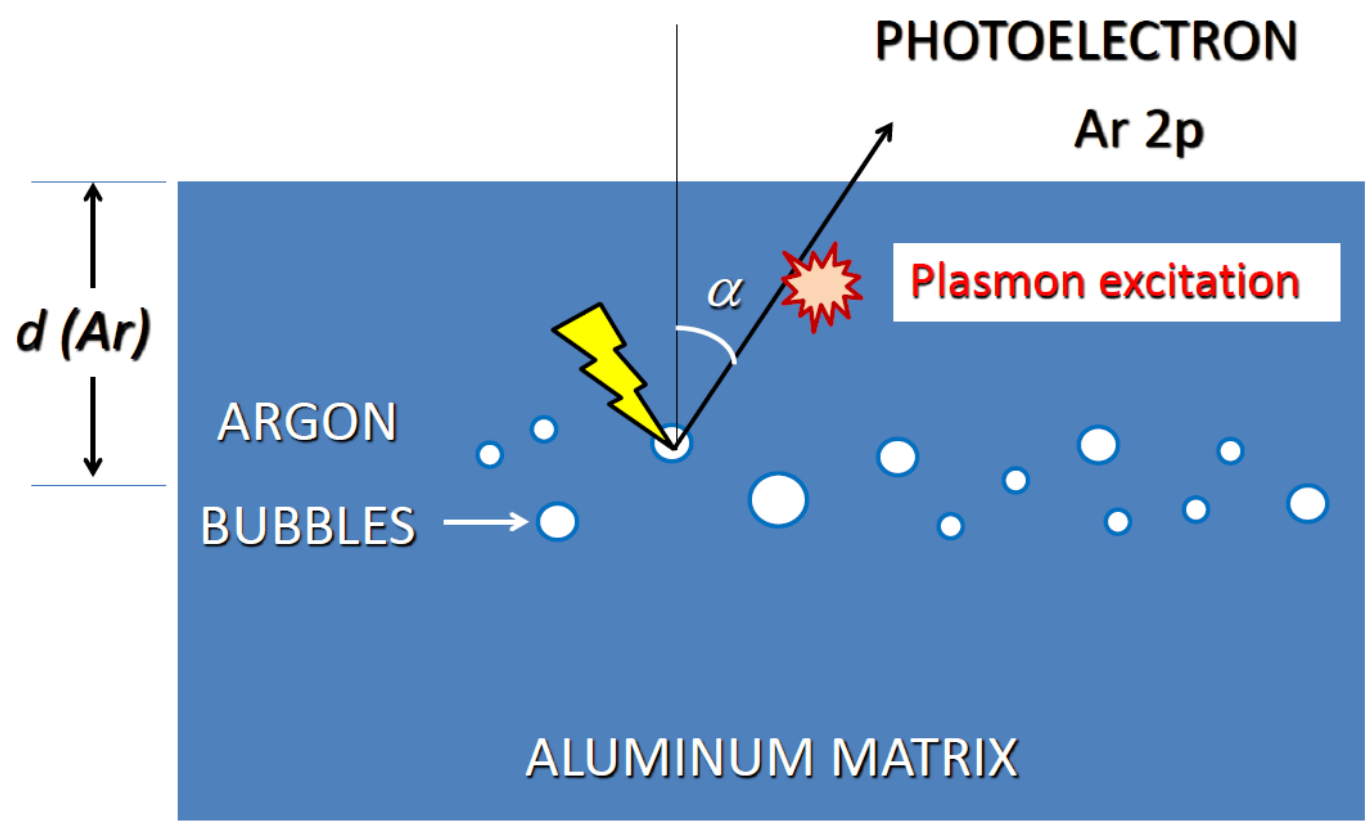


Figure 2

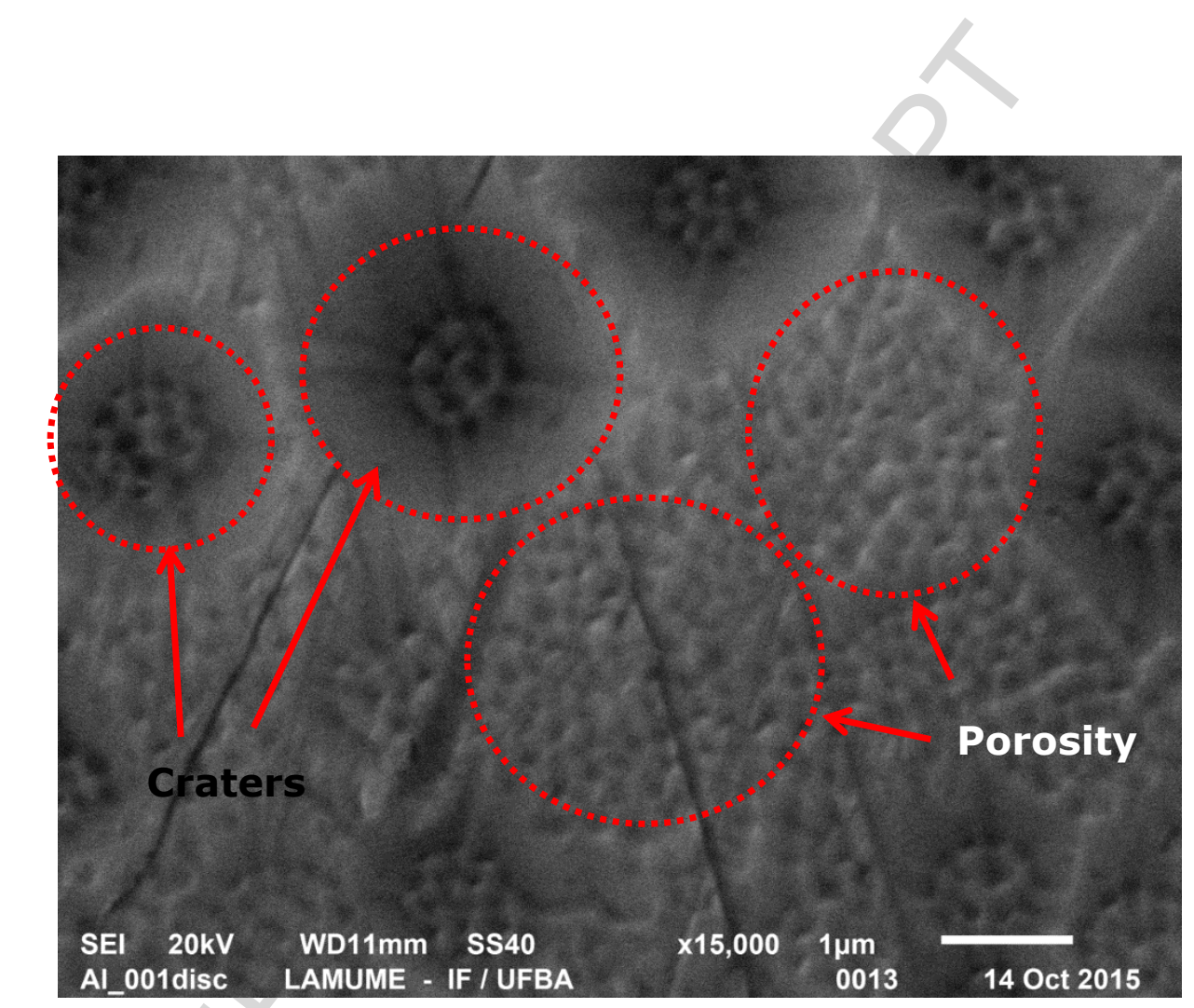


Figure 3

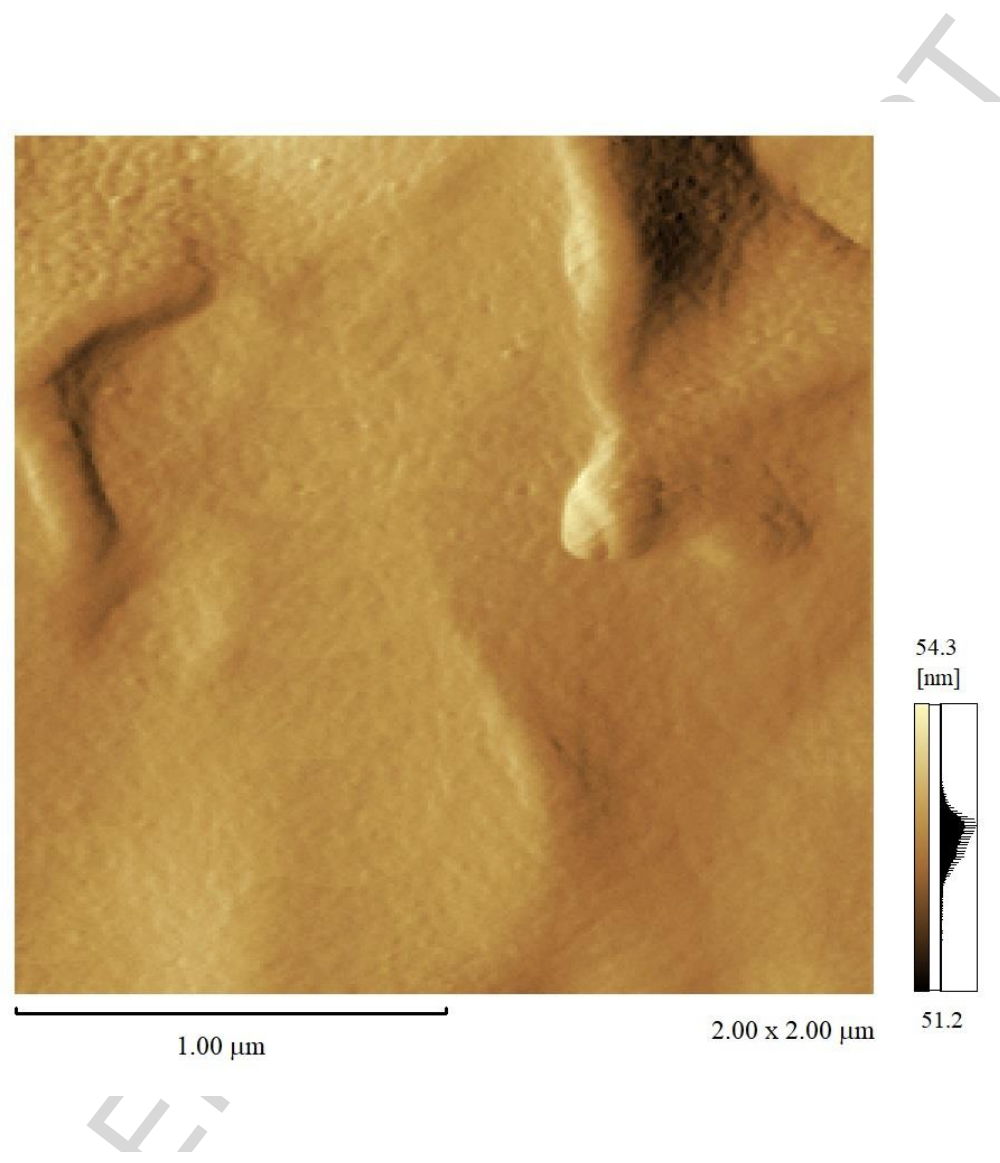




\section{Figure 4}
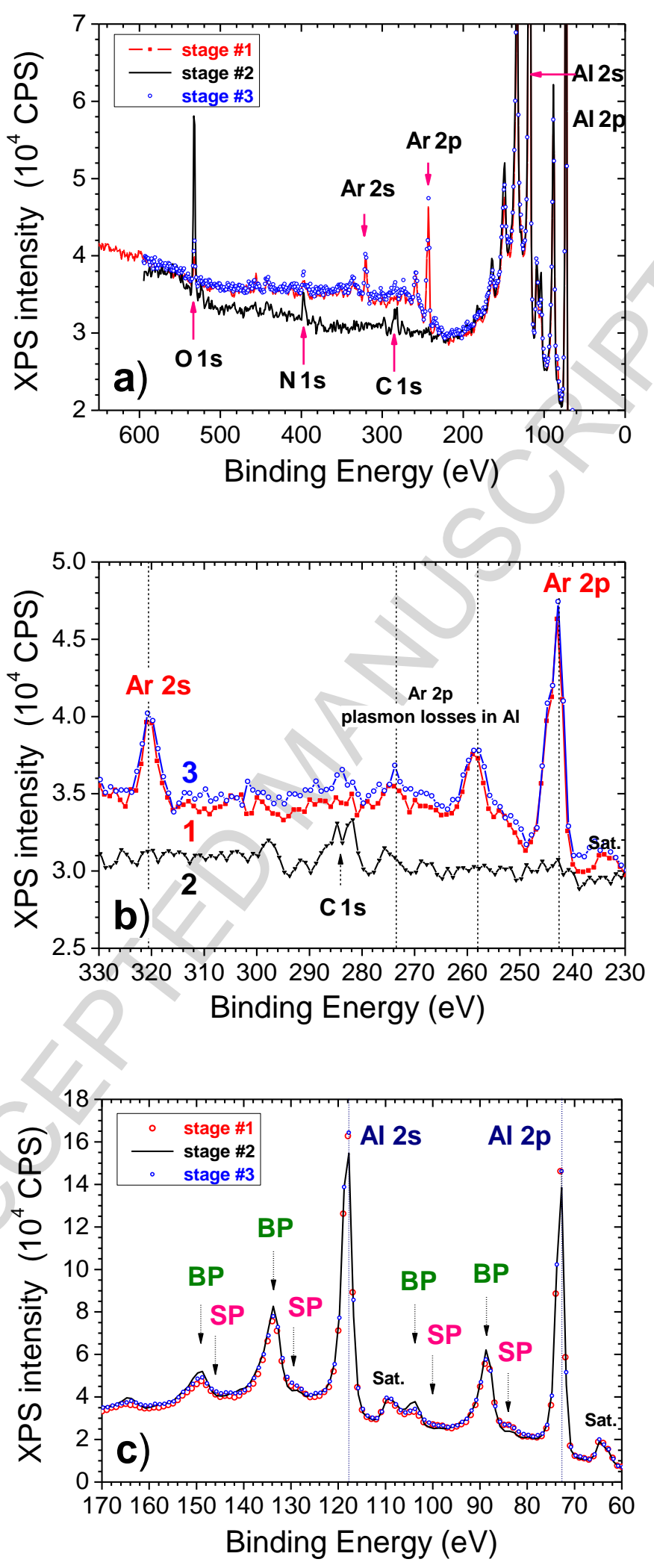


\section{Figure 5}

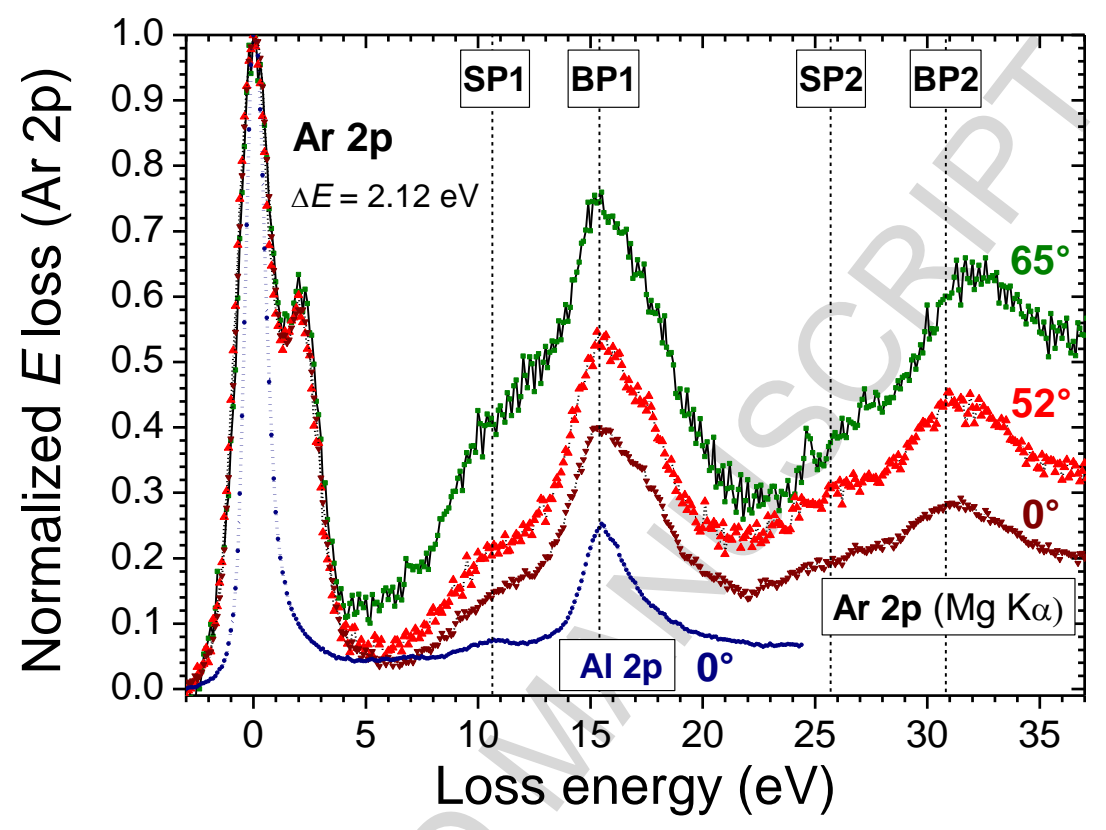




\section{Figure 6}

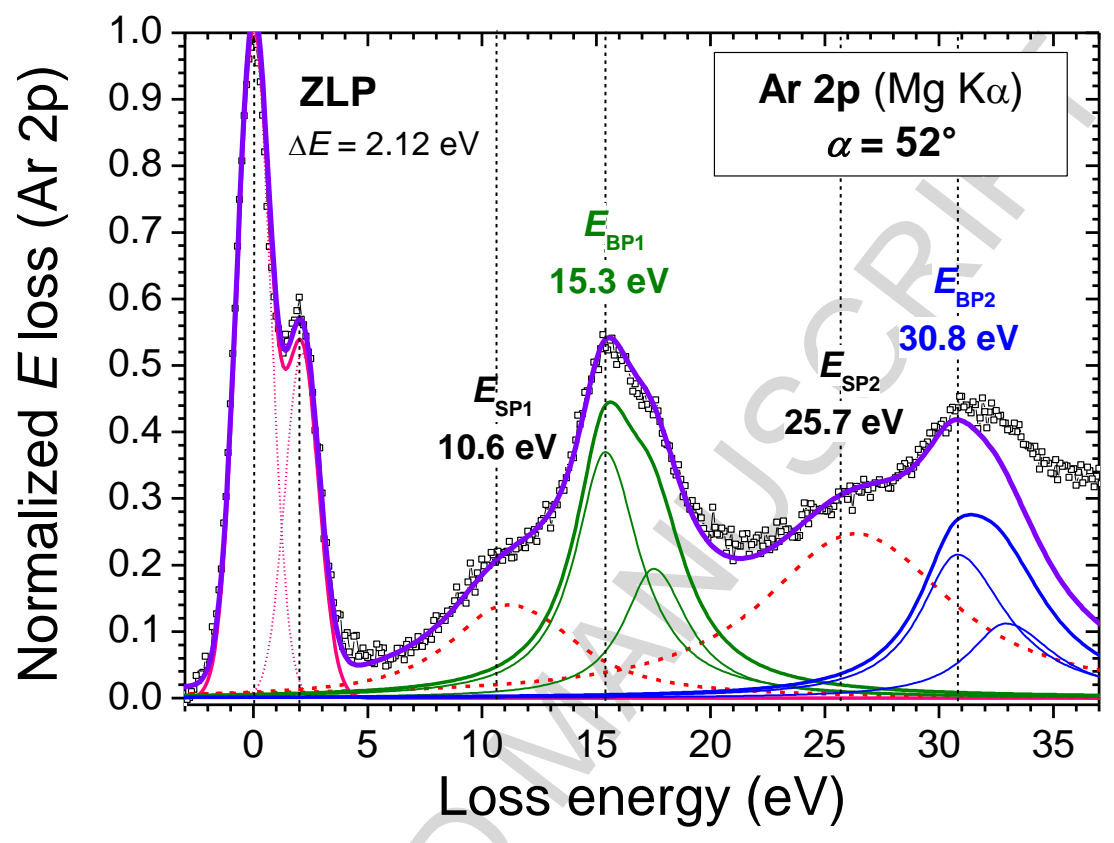


Figure 7
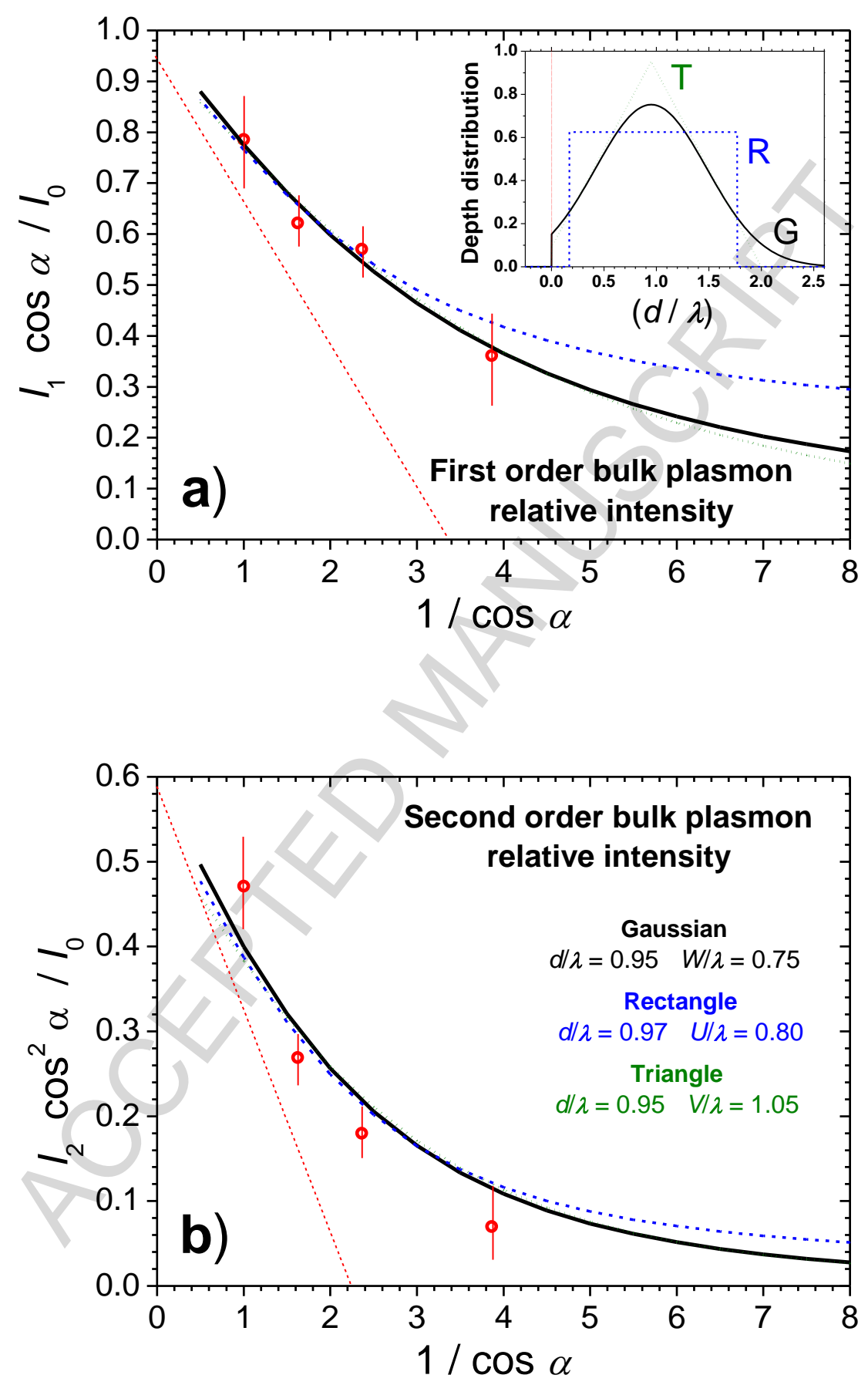